\title{
Hamiltonian Dynamical Systems
}

\author{
Heinz Hanßmann
}

Aachen, 2007 



\section{Contents}

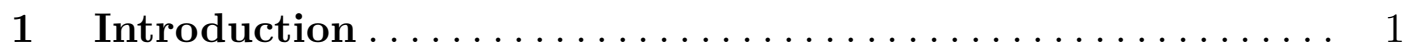

1.1 Newtonian mechanics . . . . . . . . . . . . . . . . . . . . 3

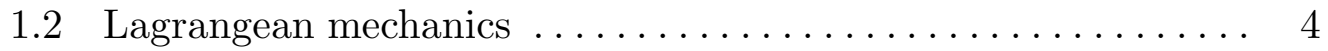

1.3 Hamiltonian mechanics...................... 5

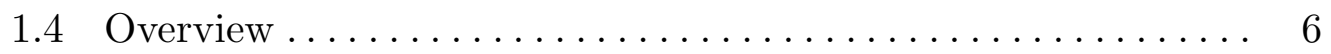

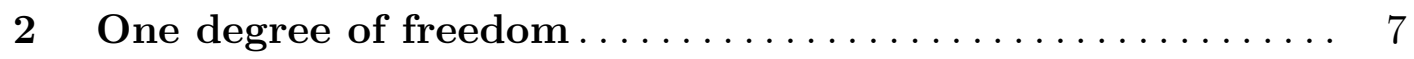

2.1 The harmonic oscillator $\ldots \ldots \ldots \ldots \ldots \ldots \ldots \ldots \ldots \ldots \ldots \ldots \ldots \ldots \ldots$

2.2 An anharmonic oscillator .................. 9

2.3 The mathematical pendulum ................ 11

3 Hamiltonian systems on the sphere $\boldsymbol{S}^{\mathbf{2}} \ldots \ldots \ldots \ldots \ldots$

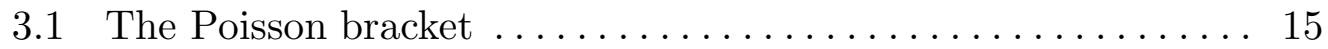

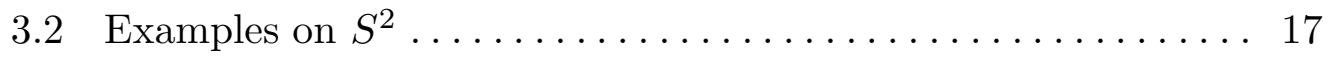

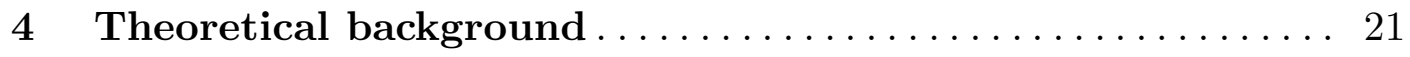

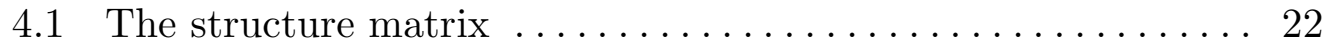

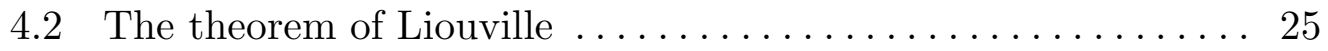

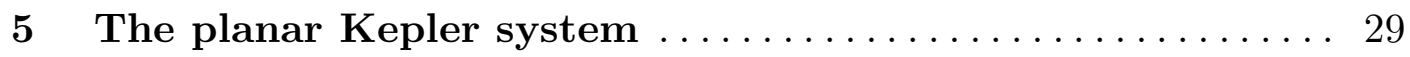

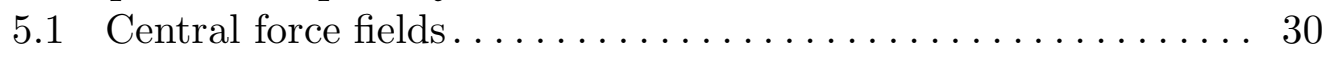

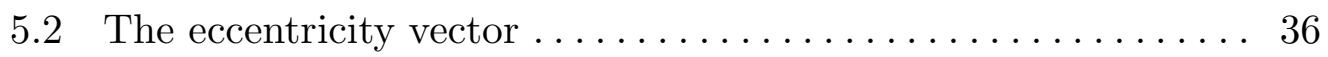

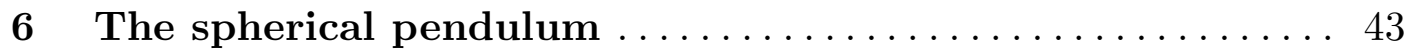

6.1 Dirac brackets ....................... 44

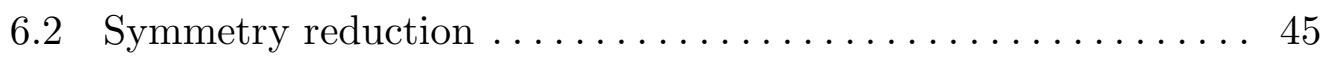

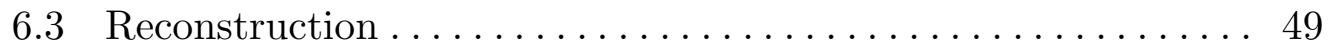

6.4 Action angle variables................... 52 
VIII Contents

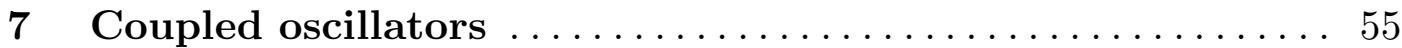

7.1 The Krein collision ............................ 58

7.2 Normalization... . . . . . . . . . . . . . . . . . . . . . . . . 60

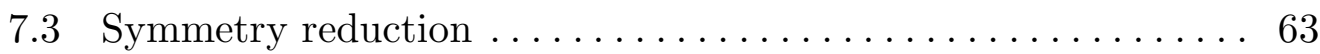

7.4 The Hamiltonian Hopf bifurcation . . . . . . . . . . . . . . . 64

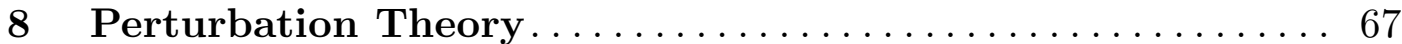

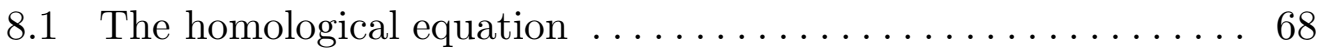

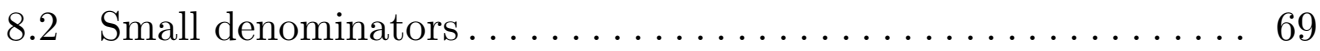

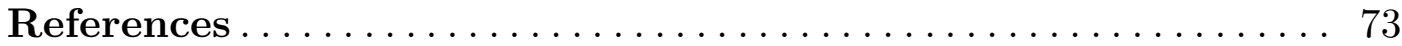




\section{Introduction}

A dynamical system on an open subset $\mathcal{P} \subseteq \mathbb{R}^{m}$ describes the time evolution of the various states $z \in \mathcal{P}$. When this description includes both (the complete) past and future this leads to a group action

$$
\begin{aligned}
\varphi: \mathbb{R} \times \mathcal{P} & \longrightarrow \mathcal{P} \\
(t, z) & \mapsto \varphi_{t}(z)
\end{aligned}
$$

of the time axis $\mathbb{R}$ on the phase space $\mathcal{P}$, i.e.

$$
\varphi_{0}=\mathrm{id} \quad \text { and } \quad \bigwedge_{s, t \in \mathbb{R}} \varphi_{s} \circ \varphi_{t}=\varphi_{s+t}
$$

This implies $\varphi_{t} \circ \varphi_{s}=\varphi_{t+s}=\varphi_{s+t}=\varphi_{s} \circ \varphi_{t}$ for all times $s, t \in \mathbb{R}$ and $\varphi_{t}^{-1}=\varphi_{-t}$ for the inverse evolution. We assume (1.1) to be smooth.

For every function $f \in C^{\infty}(\mathcal{P})$ we can consider the time evolution $f \circ \varphi_{t} \in$ $C^{\infty}(\mathcal{P})$ and in particular the time derivative

$$
\dot{f}=\left.\frac{\mathrm{d}}{\mathrm{d} t} f \circ \varphi_{t}\right|_{t=0} .
$$

The mapping

$$
\begin{array}{ccc}
C^{\infty}(\mathcal{P}) & \longrightarrow & C^{\infty}(\mathcal{P}) \\
f & \mapsto & \dot{f}
\end{array}
$$

is a derivation, i.e. a linear mapping that satisfies the Leibniz rule when applied to the product of two functions. The simplest examples of a derivation are the partial derivatives along the co-ordinate axes and the chain rule shows that

$$
\dot{f}=\sum_{i=1}^{m} \frac{\partial f}{\partial z_{i}} \frac{\mathrm{d} z_{i}}{\mathrm{~d} t}=\left(\sum_{i=1}^{m} \dot{z}_{i} \frac{\partial}{\partial z_{i}}\right) f
$$

is merely a linear combination of these, with coefficients in $C^{\infty}(\mathcal{P})$. 
Exercise 1.1. Show that every derivation on $C^{\infty}(\mathcal{P})$ is such a linear combination of partial derivatives. Hint: consider the projections $z \mapsto z_{i}$ to the co-ordinates and the polynomial functions $z \mapsto \sum a_{k} z_{1}^{k_{1}} \cdots z_{m}^{k_{m}}$.

Hence, derivations correspond to directional derivatives

$$
\sum_{i=1}^{m} g_{i} \frac{\partial}{\partial z_{i}}
$$

with coefficient functions $g_{i} \in C^{\infty}(\mathcal{P})$. Our aim is to further relate a given derivation to a dynamical system (1.1). Equating $\dot{f}$ with (1.2) yields the system

$$
\dot{z}_{i}=g_{i}(z), \quad i=1, \ldots, m
$$

of differential equations. Thus, given $z^{0} \in \mathcal{P}$ there exists a unique solution $z: I \longrightarrow \mathcal{P}$ satisfying $z(0)=z^{0}$ and $\dot{z}(t)=g(z(t))$ for all $t \in I$. Here $I \subseteq \mathbb{R}$ is the maximal interval on which the solution passing through $z^{0}$ is defined.

Exercise 1.2. Show that the flow $\varphi:\left(t, z^{0}\right) \mapsto z(t)$ defines a group action on $\mathcal{P}$ if and only if the maximal interval $I$ coincides with all of $\mathbb{R}$ for every initial condition $z^{0}$.

Where $I$ is not all of $\mathbb{R}$ the equation

$$
\varphi\left(s, \varphi\left(t, z^{0}\right)\right)=\varphi\left(s+t, z^{0}\right)
$$

only holds true when $s, t, s+t \in I$. This is called a local group action, by definition the mapping

$$
\varphi: \bigcup_{z \in \mathcal{P}} I_{z} \times\{z\} \longrightarrow \mathcal{P}
$$

still satisfies $\varphi_{0}=\mathrm{id}$. On the other hand, replacing (1.1) by a local group action still yields the derivation $f \mapsto \dot{f}$ and one still speaks of a dynamical system.

Next to the algebraic aspect of defining a derivation the system of differential equations (1.3) can also be given a geometric interpretation. The assignment of a vector $g(z) \in \mathbb{R}^{m}$ at each point $z \in \mathcal{P} \subseteq \mathbb{R}^{m}$ defines a vector field and the solution curves are tangent to each vector at each point. The directional derivative (1.2) measures how much a function $f \in C^{\infty}(\mathcal{P})$ changes along these vectors and hence along the solution curves, in particular $\dot{f}=0$ if and only if $f$ is invariant under the (local) group action.

Exercise 1.3. Show that a group action $F: \mathbb{Z} \times \mathcal{P} \longrightarrow \mathcal{P}$ is equivalent to iterating an invertible mapping on $\mathcal{P}$. What does iterating a non-invertible mapping amount to? 


\subsection{Newtonian mechanics}

There are various approaches to mechanical problems. Probably the best known is based on Newton's axioms and leads to the (differential) equation

$$
m \ddot{x}=F
$$

for the motion of a body with (fixed) mass $m$. Here $x \in \mathbb{R}^{3}$ denotes the position of the body, so $\dot{x}=\frac{\mathrm{d} x}{\mathrm{~d} t}$ is its velocity and $\ddot{x}=\frac{\mathrm{d}^{2} x}{\mathrm{~d} t^{2}}$ its acceleration. Once the force $F$ on the right hand side of (1.4) is specified and both position $x\left(t_{0}\right)=x_{0}$ and velocity $\dot{x}\left(t_{0}\right)=v_{0}$ at time $t_{0}$ are exactly known, the position $x(t)$ is determined for all ${ }^{1}$ times. This solution and its derivatives $\dot{x}(t)$ and $\ddot{x}(t)$ satisfy

$$
m \ddot{x}(t)=F(t, x(t), \dot{x}(t))
$$

at all times, so it would in fact suffice to specify the vector $F$ only along the path $(t, x(t), \dot{x}(t))$. However, we are interested in the solutions to all initial conditions, so the complete time-dependent vector field

$$
F: \mathbb{R} \times \mathbb{R}^{3} \times \mathbb{R}^{3} \longrightarrow \mathbb{R}^{3}
$$

should be specified ( $F$ may in principle also depend on extra parameters, that take different values for different bodies).

Note that the equation of motion (1.4) is a second order equation, this is rephrasing Descartes ${ }^{2}$ principle of inertia. The Aristotelian view that all bodies tend to rest would favour a first order differential equation, and if the equation of motion were of third order, then one would have to also prescribe the initial acceleration $\ddot{x}\left(t_{0}\right)=a_{0}$ to determine the motion.

The force field $F$ is rather general and may for instance contain a friction term $-\alpha \dot{x}$. Even if $F$ is time-independent and positional, i.e. $F=F(x)$, it is still an extra assumption that the work $W(x, y)$ done by $F$ between two positions $x$ and $y$ does not depend on the path used to move from $x$ to $y$, in which case one may define the potential energy $V(x)=W\left(x, x_{0}\right)$ by choosing e.g. $x_{0}=0$ - a different choice $x_{1}$ leads to the additional constant $W\left(x_{0}, x_{1}\right)$ and has no consequences for

$$
F(x)=-\nabla V(x)
$$

For such a force field any solution $x(t)$ of (1.4) conserves the sum $T+V$ of kinetic energy $\frac{1}{2} m(\dot{x}(t))^{2}$ and potential energy $V(x(t))$.

\footnotetext{
${ }^{1}$ All times is the maximal interval given by the theorem on existence and uniqueness of differential equations, for which we need the assumption that $F$ is continuous and satisfies locally a Lipschitz condition.

2 Discours de la méthode (1637), see also Galilei's Discorsi e dimostrazioni matematiche (1638).
} 
Introducing $p:=m \dot{x}$ the second order equation (1.4) can be written as the system

$$
\begin{aligned}
\dot{x} & =\frac{1}{m} p \\
\dot{p} & =F(t, x, p)
\end{aligned}
$$

of first order equations. The dutch, english, french and german versions of Wikipedia fiercely disagree on whether this is in fact how Newton formulated his second axiom.

\subsection{Lagrangean mechanics}

The Lagrangean approach to mechanics is based on a variational principle. The underlying idea that "nature tries to minimize something" has also proven successful in other branches of physics, refraction of light may for instance be thought of as light choosing the shortest way through an optical medium.

Possible motions in Lagrangean mechanics are extremals $x(t)$ of the action functional, which in turn is defined as the integral of the Lagrangean function $L=L(t, x, v)$ along the path

$$
\gamma: t \mapsto(t, x(t), \dot{x}(t))
$$

over time. Minimizing

$$
\Phi(\gamma)=\int_{t_{0}}^{t_{1}} L(t, x(t), \dot{x}(t)) \mathrm{d} t
$$

leads to the necessary condition

$$
D \Phi(\gamma)=0
$$

where $D$ denotes the derivative with respect to all "admissible" paths $\gamma$, and declaring this condition to be sufficient yields the extremals of (1.5).

The basic theorem of the calculus of variations states that the extremals $\gamma$ of $\Phi$ are exactly those paths which satisfy the Euler-Lagrange equation

$$
\frac{\mathrm{d}}{\mathrm{d} t}\left(\nabla_{v} L\right)=\nabla_{x} L
$$

It is a co-ordinate free property of a path $\gamma$ to be an extremal of $\Phi$ whence we may choose any system $q_{1}, \ldots, q_{n}$ of "generalized" co-ordinates for the position $x$ of the mechanical system and the Euler-Lagrange equation takes the form

$$
\frac{\mathrm{d}}{\mathrm{d} t}\left(\frac{\partial L}{\partial \dot{q}_{i}}\right)=\frac{\partial L}{\partial q_{i}}, \quad i=1, \ldots, n .
$$


Note that the freedom of co-ordinate choice does not extend to the velocity $v$ of the mechanical system, on the contrary, $\dot{q}_{1}, \ldots, \dot{q}_{n}$ are directly determined from the $q_{i}$. The notational choice of letting $\dot{q}_{i}$ denote both co-ordinates and time derivatives turns out to be extremely useful in computations.

For the motion of a body with (fixed) mass $m$ in a positional force field derived from the potential $V$ the Lagrangean is the difference $L=T-V$ between kinetic energy $T=\frac{1}{2} m v^{2}$ and potential energy and the Euler-Lagrange equation reads

$$
m \ddot{x}=m \dot{v}=-\nabla V(x)
$$

as in Newtonian mechanics.

\subsection{Hamiltonian mechanics}

The Hamiltonian formulation builds the conservation of energy into the theory. For the motion of a body with (fixed) mass $m$ in a positional force field derived from the potential $V$ the Hamiltonian function is the sum $H=T+V$ of kinetic energy $T=\frac{p^{2}}{2 m}$ and potential energy. The equations of motion are

$$
\begin{aligned}
\dot{x} & =D_{p} H \\
\dot{p} & =-D_{x} H
\end{aligned}
$$

and reproduce again (1.4). However, for any other choice $H=H(x, p)$ the system (1.7) conserves the Hamiltonian as well:

$$
\dot{H}=D_{x} H \cdot \dot{x}+D_{p} H \cdot \dot{p}=0 .
$$

Thus, the vector field defined by (1.7) is tangent to the level sets of $H$. Perpendicular to these is the gradient vector field, which points in the direction of maximal growth of $H$.

Exercise 1.4. Show that every system of $n$ differential equations can be completed to a Hamiltonian system on $\mathbb{R}^{2 n}$.

Exercise 1.5. Show that the linearization of a Hamiltonian system is again Hamiltonian. How are the corresponding Hamiltonian functions related to each other?

Exercise 1.6. Let $H(x, p)=\frac{p^{2}}{2 m}+V(x)$ be the energy function, where $V: \mathbb{R} \longrightarrow \mathbb{R}$ is the potential energy. Compute the Legendre transformation

$$
L(x, v):=\sup _{p \in \mathbb{R}}(v \cdot p-H(x, p))
$$

and clarify the situation with a figure. The function $L$ obtained this way is the Lagrangean function of the system. Show that Hamilton's equations (1.7) turn into (1.6). 
Exercise 1.7. Show that every second order differential equation $\ddot{x}=f(x)$ in $x \in \mathbb{R}$ defines a Hamiltonian system. How can this be generalized to higher dimensions?

\subsection{Overview}

As we have seen, the Newtonian, Lagrangean and Hamiltonian approaches are equivalent for the motion of a body with fixed mass in a positional conservative force field. It turns out that this equivalence extends to all situations in which at least two of these approaches are applicable. A priori it was not evident that the causal formulations behind the equations of motion (1.4) and (1.7) yield the same description of mechanics as the teleological formulation to minimize (1.5).

Given a mechanical system, it is a non-trivial task to write down the Lagrangean or the Hamiltonian. In the sequel we concentrate on the case that the Hamiltonian consists of the (total) energy of the system. Then the equations of motion (1.7) follow immediately and our aim is to study the resulting dynamics. To this we adhere to the following outline.

(i) Systems with one degree of freedom.

(ii) Theoretical background.

(iii) Systems with two degrees of freedom.

(iv) General results.

(v) Systems with three degrees of freedom.

Emphasis will be on (iii) and we will see that the complete solution of arbitrary such systems is already beyond our present means. The methods by which at least something can be said motivate $(i v)$, similarly the first "theoretical part" $(i i)$ is motivated by $(i)$. In fact, the theory will always be delayed until it is actually needed. 


\section{One degree of freedom}

A particle/mass point has one degree of freedom if it can only move in one direction, the $x$-direction. In the simplest case, that of (force)-free motion, the potential energy vanishes and the particle moves subject only to its own inertia, with kinetic energy

$$
H(x, p)=\frac{p^{2}}{2 m}
$$

(with momentum $p=m v$, so this rephrases indeed $T=\frac{1}{2} m v^{2}$ ). Hamilton's equations (1.7) then read

$$
\begin{aligned}
& \dot{x}=\frac{\partial H}{\partial p}=\frac{1}{m} p \quad(\text { so indeed } \dot{x}=v) \\
& \dot{p}=-\frac{\partial H}{\partial x}=0
\end{aligned}
$$

with the second equation expressing conservation of linear momentum - in the absence of force. For given initial conditions $\left(x_{0}, p_{0}\right)$ these equations can easily be solved,

$$
x(t)=x_{0}+\frac{p_{0}}{m} t, \quad p(t) \equiv p_{0},
$$

the particle moves with constant speed on a straight line.

From now on we change notation and use $y$ instead of $p$, this enables us to work with co-ordinate changes $(x, y) \mapsto(q, p)$.

Exercise 2.1. Show that $x \mapsto x+\xi$ is a symmetry of the free linear motion and explain that the corresponding group action coincides with the flow of Hamilton's equations.

Exercise 2.2. Give a necessary condition for the dynamical system

$$
\begin{aligned}
\dot{x} & =f(x, y) \\
\dot{y} & =g(x, y)
\end{aligned}
$$

on the open set $U \subseteq \mathbb{R}^{2}$ to be Hamiltonian. Is the condition also sufficient? 


\subsection{The harmonic oscillator}

Let the particle now move in a quadratic potential with minimum at $x=0$. Putting the mass $m=1$ the Hamiltonian takes the form

$$
H(x, y)=\frac{1}{2} y^{2}+\frac{\omega^{2}}{2} x^{2}
$$

with equations of motion

$$
\begin{aligned}
& \dot{x}=y \\
& \dot{y}=-\omega^{2} x .
\end{aligned}
$$

For initial conditions $\left(x_{0}, y_{0}\right)$ the solution reads

$$
\begin{aligned}
& x(t)=x_{0} \cos \omega t+\frac{y_{0}}{\omega} \sin \omega t \\
& y(t)=-\omega x_{0} \sin \omega t+y_{0} \cos \omega t .
\end{aligned}
$$

These are ellipses in the $(x, y)$-plane, changing co-ordinates to

$$
q=\sqrt{\omega} x \text { and } p=\frac{y}{\sqrt{\omega}}
$$

we obtain the circular solutions of the system

$$
\frac{\mathrm{d}}{\mathrm{d} t}\left(\begin{array}{c}
q \\
p
\end{array}\right)=\left(\begin{array}{cc}
0 & \omega \\
-\omega & 0
\end{array}\right)\left(\begin{array}{l}
q \\
p
\end{array}\right) .
$$

This second example is more "typical" for the systems we are going to study than the free particle was, since the motion is bounded.

Exercise 2.3. Let

$$
\begin{aligned}
& \dot{q}=\omega p \\
& \dot{p}=-\omega q, \quad \omega>0
\end{aligned}
$$

be a harmonic oscillator. Transform the system to "action angle variables" $\varphi, I$ according to

$$
q=\sqrt{\frac{I}{\pi}} \cos 2 \pi \varphi, \quad p=\sqrt{\frac{I}{\pi}} \sin 2 \pi \varphi
$$

and conclude that the flows with different $\omega$ are equivalent to each other, having the same orbits.

Exercise 2.4. What are the possible types of linear Hamiltonian systems with one degree of freedom? How do the corresponding Hamiltonian functions look like? Which of the occurring equilibria are structurally stable - and in what sense? 


\subsection{An anharmonic oscillator}

For a fourth order potential, still with a minimum at $x=0$, the Hamiltonian reads

$$
H(x, y)=\frac{1}{2} y^{2}+\frac{1}{24} x^{4}
$$

with equations of motion

$$
\begin{aligned}
\dot{x} & =y \\
\dot{y} & =-\frac{1}{6} x^{3} .
\end{aligned}
$$

The phase portrait can be obtained from the graph of the potential energy, see Fig. ?. Since energy is conserved, the potential energy at $y=0$ is transformed into kinetic energy at $x=0$ and vice versa.

Exercise 2.5. Determine the phase portrait for a 1-dimensional particle moving in the potential

$$
V(x)=-x^{2} \cdot \mathrm{e}^{-x}
$$

Exercise 2.6. Consider a function $H: \mathbb{R}^{2} \longrightarrow \mathbb{R}$, of the form $H(x, y)=$ $\frac{1}{2} y^{2}+V(x)$. For several shapes of the graph of $V$, sketch the phase portrait of $X_{H}$. How do the integral curves intersect the $x$-axis? In particular consider cases where $V$ has maxima, minima or a horizontal asymptote.

Perturb now to $H(x, y)=\frac{1}{2} y^{2}+\frac{1}{24} x^{4}+\frac{\lambda}{2} x^{2}$ with equations of motion

$$
\begin{aligned}
& \dot{x}=y \\
& \dot{y}=-\frac{1}{6} x^{3}-\lambda x
\end{aligned}
$$

and phase portraits given in Fig. ?. Under variation of $\lambda$ a Hamiltonian pitchfork bifurcation takes place. Correspondingly, the linearization of the anharmonic oscillator (2.1) in the origin is given by the matrix

$$
\left(\begin{array}{ll}
0 & 1 \\
0 & 0
\end{array}\right)
$$

whence this equilibrium is parabolic.

Exercise 2.7. Let $a_{0}$ be a minimum of the energy, whence(?!) the motion for slightly larger energy values $E$ is periodic with period $T(E)$. Determine the limit

$$
T_{0}:=\lim _{E \rightarrow E\left(a_{0}\right)} T(E)
$$


The typical way in which a parabolic equilibrium bifurcates is the centresaddle bifurcation. Here the Hamiltonian reads

$$
H(x, y)=\frac{a}{2} y^{2}+\frac{b}{6} x^{3}+c \lambda x
$$

where $a, b, c \in \mathbb{R}$ are nonzero constants. For instance, when $a=b=c=1$ this leads to the phase portraits given in Fig. ?.

Note that this is a completely different unfolding of the parabolic equilibrium at the origin. A closer look at the phase portraits and in particular at the Hamiltonian function of the Hamiltonian pitchfork bifurcation reveals the symmetry $x \mapsto-x$. This suggests to add the non-symmetric term $\mu x$.

Exercise 2.8. Determine the bifurcation diagram of the family

$$
H_{\lambda, \mu}(x, y)=\frac{1}{2} y^{2}+\frac{1}{24} x^{4}+\frac{\lambda}{2} x^{2}+\mu x
$$

of Hamiltonian systems.

Singularity theory allows to prove that upon adding further "small" terms to $H_{\lambda, \mu}$ no additional phase portraits are generated. Up to equivalence (i.e. qualitatively) $H_{\lambda, \mu}$ contains all possible unfoldings of the anharmonic oscillator (2.1), one also speaks of a versal unfolding. Similarly, the centre-saddle bifurcation is stable 1-parameter family.

Exercise 2.9. Analyse the family

$$
H_{\lambda}(x, y)=\frac{1}{2} y^{2}+\frac{1}{6} x^{3}+\frac{\lambda}{2} x^{2}
$$

of Hamiltonian systems.

Up to now all Hamiltonians were invariant with respect to $y \mapsto-y$. This reversibility (reflecting the phase portrait about the $x$-axis and simultaneously reversing the flow of time leaves the system invariant) is built into simple mechanical systems with positional potential energy $V=V(x)$ and kinetic energy $T=\frac{1}{2} y^{2}$. Note that adding an unfolding term $\nu y$ does not lead to qualitatively new phenomena since one may simply apply the co-ordinate transformation $(x, y) \mapsto(x, y-\nu)$ to the perturbed system.

Exercise 2.10. Consider the Hamiltonian $H(x, y)=x y^{2}-\frac{1}{3} x^{3}$. Use monomials like $x, \frac{1}{2} x^{2}$ or $\frac{1}{2} y^{2}$ to unfold preserving the reversibility and monomials like $y$ or $x y$ to break the reversibility. Conclude that the latter does lead to qualitatively new phenomena. Can you guess the minimal number of parameters of a versal unfolding? 


\subsection{The mathematical pendulum}

The mathematical idealization of a pendulum is a mass point fixed to a massless rod (not a string, so the pendulum can also "turn over the top"). We use the positional variable $x \in \mathbb{R}$ to measure the angle from the vertical, whence the height of the pendulum (i.e. of the mass point) is given by $V(x)=-\cos x$ and the energy reads

$$
H(x, y)=\frac{1}{2} y^{2}-\cos x
$$

From the graph of the potential energy we obtain the phase portraits in Fig. ? while the equations of motion obtained from $H$ are given by

$$
\begin{aligned}
& \dot{x}=y \\
& \dot{y}=-\sin x .
\end{aligned}
$$

The equilibria are $(x, y)=(k \pi, 0), k \in \mathbb{Z}$ and have linearization

$$
\left(\begin{array}{cr}
0 & 1 \\
-\cos k \pi & 0
\end{array}\right)=\left(\begin{array}{cc}
0 & 1 \\
-(-1)^{k} & 0
\end{array}\right)
$$

with determinant $(-1)^{k}$, whence equilibria with even $k$ are centres and equilibria with odd $k$ are saddles. Note that phase portrait, Hamiltonian function, equations of motion, ... are $2 \pi$-periodic in $x$ - the pendulum does not know the difference between the position of the mass point at an angle $x$ and "that same angle" $x+2 k \pi$ after several full rotations. It is therefore helpful to take the co-ordinate $x \bmod 2 \pi$ and pass to the phase space $S^{1} \times \mathbb{R}$, see Fig. ?. On the resulting cylinder the "rotating motions" over the top are periodic.

For any potential energy $V: S^{1} \longrightarrow \mathbb{R}$ the Hamiltonian $H=T+V$ with kinetic energy $T=\frac{1}{2} y^{2}$ defines equations of motion

$$
\begin{aligned}
\dot{x} & =y \\
\dot{y} & =-V^{\prime}(x)
\end{aligned}
$$

on $S^{1} \times \mathbb{R}$; the corresponding flow may be computed directly on the phase space or first on $\mathbb{R}^{2}$ and then projected $\bmod 2 \pi$ in the first component.

Exercise 2.11. Consider the Hamiltonian function $H(x, y)=\frac{1}{2} y^{2}-\cos x$ of the pendulum. For $|z|<1$ we consider the level set $H^{-1}(z)$. What is the amplitude of oscillation in this level? If $T(z)$ denotes the period of oscillation in this level, then give an explicit integral expression for this. Determine $\lim _{z \rightarrow-1} T(z)$ and $\lim _{z \rightarrow+1} T(z)$.

Exercise 2.12. Analyse the dynamics of the rotating pendulum $\ddot{x}=M-\sin x$ in dependence of $M$. 
Exercise 2.13. Let $H(x, y)=\frac{1}{2} y^{2}-V(x)$ be the Hamiltonian of a 1dimensional particle with mass $m=1$ moving in the potential $V$. Describe the time parametrisations of the trajectories $H=h$ in terms of the indefinite integral

$$
\int \frac{\mathrm{d} x}{\sqrt{2(h-V(x))}} .
$$

Exercise 2.14. Let $H \in C^{\infty}(\mathcal{P})$ be a Hamiltonian and $\left.\beta: \mathcal{P} \longrightarrow\right] 0, \infty[$ a smooth function. Define another Hamiltonian $K \in C^{\infty}(\mathcal{P})$ by $K(z):=$ $\beta(z) \cdot(H(z)-h)$ for all $z \in \mathcal{P}$. Show that the trajectories of $X_{K}$ on $K^{-1}(0)$ are related to the trajectories of $X_{H}$ on $H^{-1}(0)$ by means of the orientation preserving re-parametrisation $\frac{\mathrm{d} \tau}{\mathrm{d} t}=\frac{1}{\beta}$ of time. 


\section{Hamiltonian systems on the sphere $S^{2}$}

Next to $x \bmod 2 \pi$ we could also work with $y \bmod 2 \pi$ and study Hamiltonian systems on the 2 -torus $S^{1} \times S^{1}$. For such a one-degree-of-freedom system there is no kinetic energy $T=\frac{1}{2} y^{2}$ and the variable $y$ loses its interpretation of being the velocity or momentum of the particle with position $x$.

This is even more true for Hamiltonian systems on $S^{2}$ which have the additional problem that it is impossible to globally define a co-ordinate system, at least one point has to be discarded.

Discarding two points on $S^{2}=\left\{(x, y, z) \in \mathbb{R}^{3}: x^{2}+y^{2}+z^{2}=1\right\}$, e.g. the north pole $z=1$ and the south pole $z=-1$, one can define cylindrical co-ordinates $p=z$ and $q \in S^{1}$ by means of

$$
\cos q=\frac{x}{\sqrt{1-z^{2}}}, \quad \sin q=\frac{y}{\sqrt{1-z^{2}}} .
$$

The equations of motion still read

$$
\begin{aligned}
& \dot{q}=\frac{\partial H}{\partial p} \\
& \dot{p}=-\frac{\partial H}{\partial q}
\end{aligned}
$$

as we have in fact brought the situation back to $S^{1} \times \mathbb{R}$.

While it is possible to treat Hamiltonian dynamical systems on $S^{2}$ in this way, switching to cylindrical co-ordinates along e.g. the $y$-axis when motions passing through north or south pole are encountered, this approach is less satisfactory.

1. The truly global questions are difficult to attack.

2. Also for local problems this is impractical.

We therefore prefer to work globally on phase spaces that are manifolds. 
Exercise 3.1. For $H \in C^{\infty}\left(\mathbb{R}^{3}\right)$ define the equations of motion

$$
\frac{\mathrm{d}}{\mathrm{d} t}\left(\begin{array}{c}
x \\
y \\
z
\end{array}\right)=\left(\begin{array}{c}
\frac{\partial H}{\partial x} \\
\frac{\partial H}{\partial y} \\
\frac{\partial H}{\partial z}
\end{array}\right) \times\left(\begin{array}{c}
x \\
y \\
z
\end{array}\right) .
$$

Show that $H$ is a constant of motion. Find a second constant of motion. What can be said about those points where both functions have linear dependent gradients?

Definition 3.1. Let $M$ be a metric space. A chart on $M$ is a homeomorphism $\varphi: U \longrightarrow V$ between an open set $U \subseteq M$ and an open set $V \subseteq \mathbb{R}^{m}$. A set of charts on $M$ forms an atlas if

(i) every point $x \in M$ lies in the source of at least one chart in the atlas;

(ii) the chart changes $\varphi_{2} \circ \varphi_{1}^{-1}: \varphi_{1}\left(U_{1} \cap U_{2}\right) \subseteq V_{1} \longrightarrow \varphi_{2}\left(U_{1} \cap U_{2}\right) \subseteq V_{2}$ are differentiable.

Singling out an atlas $A$ makes $M$ a differentiable manifold. We then say that a function $f: M \longrightarrow \mathbb{R}$ is differentiable if

$$
\bigwedge_{\varphi \in A} f \circ \varphi^{-1}: V \longrightarrow \mathbb{R} \text { is differentiable. }
$$

Two atlasses are called compatible if their union is again an atlas. The union of all atlasses compatible with $A$ is the maximal atlas of the differentiable manifold $M$.

Examples are $\mathbb{R}^{m}$ itself, the cylinder $S^{1} \times \mathbb{R}$, the spheres $S^{m}$ and the tori $\mathbb{T}^{m}=$ $\mathbb{R}^{m} / \mathbb{Z}^{m}$. We like our manifolds to be as smooth as needed, i.e. $C^{k}$ with $k \in \mathbb{N}$ sufficiently high, and work from now on for simplicity with $C^{\infty}$ manifolds.

Exercise 3.2. Show that $\mathbb{T}^{m}$ is diffeomorphic with $S^{1} \times \ldots \times S^{1}$.

The atlas allows to transport a concept from open subsets $V \subseteq \mathbb{R}^{m}$ to a manifold $\mathcal{P}$ if the concept is local in nature and not depending on a special choice of co-ordinates. For a dynamical system the notions of (local) group action and of a derivation $C^{\infty}(\mathcal{P}) \longrightarrow C^{\infty}(\mathcal{P})$ are already globally defined. In every chart the considerations from the introduction remain valid, in particular

$$
\dot{f}=\sum_{i=1}^{m} \dot{z}_{i} \frac{\partial f}{\partial z_{i}}
$$

for the directional derivative. The corresponding locally defined (and coordinate depending) vector fields can be given a global meaning. To this end call two curves $\gamma, \eta: I \longrightarrow \mathcal{P}$ with $\gamma(0)=\eta(0)=: z^{0}$ equivalent (at $z^{0}$ ) if the time derivatives of $\gamma$ and $\eta$ at 0 coincide in one (and hence every) chart 
around $z^{0}$. The equivalence classes of this equivalence relation are the tangent vectors (at $z^{0}$ ), and a vector field assigns to each $z^{0} \in \mathcal{P}$ a tangent vector at $z^{0}$. In each chart this amounts to a system of differential equations, and the solution curves have at each point a time derivative coinciding with the prescribed tangent vector (i.e. the time derivative lies in this equivalence class). The resulting flow is (again) a local group action.

Exercise 3.3. Show that on a compact manifold $\mathcal{P}$ each vector field determines a group action (of all of $\mathbb{R}$ ) on $\mathcal{P}$. Hint: consider the maximal time interval $I \subseteq \mathbb{R}$ of a given solution curve.

To define the Lie bracket $[X, Y]$ of two vector fields consider these as derivations $X, Y: C^{\infty}(\mathcal{P}) \longrightarrow C^{\infty}(\mathcal{P})$ and put

$$
[X, Y](f):=X(Y(f))-Y(X(f)) .
$$

Exercise 3.4. Compute the Lie bracket $\left[\frac{\partial}{\partial z_{i}}, \frac{\partial}{\partial z_{j}}\right]$ of two partial derivatives along the co-ordinate axes in $\mathbb{R}^{m}$.

Exercise 3.5. Let $\varphi, \psi$ be the (globally defined) flows of two vector fields $X, Y$ on a manifold $\mathcal{P}$ and assume that

$$
\bigwedge_{s, t \in \mathbb{R}} \varphi_{t} \circ \psi_{s}=\psi_{s} \circ \varphi_{t},
$$

i.e. the flows commute. Conclude that then the Lie bracket $[X, Y]$ vanishes identically. Can you also prove the converse?

In this sense the Lie bracket of two vector fields measures to what extent the corresponding flows commute.

Exercise 3.6. Show that the "natural mechanical system" $H=\frac{1}{2} y^{2}+V(x)$ has a globally defined flow in the following cases.

1. The potential energie $V$ is everywhere positive.

2. The phase space is $S^{1} \times \mathbb{R}$.

In how far is the first condition physically relevant?

\subsection{The Poisson bracket}

To write down the equations of motion without resorting to (canonical) coordinates we work with Poisson brackets.

Definition 3.2. Let $\mathcal{P}$ be a differentiable manifold. A mapping

$$
\{,\}: C^{\infty}(\mathcal{P}) \times C^{\infty}(\mathcal{P}) \longrightarrow C^{\infty}(\mathcal{P})
$$

is called a Poisson bracket if it satisfies the following conditions. 
(i) $\{$,$\} is bilinear, i.e. linear in both arguments.$

(ii) $\{$,$\} is alternating, i.e. \bigwedge_{f \in C^{\infty}(\mathcal{P})}\{f, f\}=0$.

(iii) $\{$,$\} satisfies the Leibniz rule$

$$
\bigwedge_{h \in C^{\infty}(\mathcal{P})}\{f \cdot g, h\}=f \cdot\{g, h\}+\{f, h\} \cdot g .
$$

(iv) $\{$,$\} satisfies the Jacobi identity$

$$
\bigwedge_{f, g, h \in C^{\infty}(\mathcal{P})}\{\{f, g\}, h\}=\{f,\{g, h\}\}+\{\{f, h\}, g\} .
$$

We then call $(\mathcal{P},\{\}$,$) a Poisson manifold.$

The Leibniz rule gives $\{$,$\} the character of a derivative (if one fixes one of$ the arguments, see below) and correspondingly Hamilton's equations become $\frac{\mathrm{d}}{\mathrm{d} t} f=\dot{f}=\{f, H\}$ on a Poisson manifold.

Exercise 3.7. Show that a Poisson bracket is anti-symmetric, i.e.

$$
\bigwedge_{f, g \in C^{\infty}(\mathcal{P})}\{g, f\}=-\{f, g\}
$$

and yields the vanishing sum of cyclic permutations

$$
\bigwedge_{f, g, h \in C^{\infty}(\mathcal{P})}\{\{f, g\}, h\}+\{\{g, h\}, f\}+\{\{h, f\}, g\}=0
$$

(this is often the form in which Jacobi's identity is stated).

The Leibniz rule expresses that the mapping $f \mapsto\{f, H\}$ is a derivation, as the partial derivative $\frac{\partial}{\partial z}$ along the co-ordinate axis in a chart of $\mathcal{P}$ is a derivation, a linear mapping satisfying the Leibniz rule. This makes $f \mapsto\{f, H\}$ a vector field on $\mathcal{P}$ whence

$$
\{f, H\}(z)=\sum_{i=1}^{m} \eta_{i}(z) \frac{\partial f}{\partial z_{i}}
$$

in local co-ordinates; in a chart the partial derivatives $\frac{\partial}{\partial z_{i}}$ along the coordinate axes form a basis of each tangent space. Applying this to the coordinate function $z_{j}$ yields

$$
\left\{z_{j}, H\right\}(z)=\sum_{i=1}^{m} \eta_{i}(z) \frac{\partial z_{j}}{\partial z_{i}}=\sum_{i=1}^{m} \eta_{i}(z) \delta_{i j}=\eta_{j}(z)
$$

whence the above expansion reads

$$
\{f, H\}=\sum_{i=1}^{m}\left\{z_{i}, H\right\} \frac{\partial f}{\partial z_{i}} .
$$


Example 3.3. On $\mathbb{R}^{2}$ a Poisson bracket is determined by the value of $\{x, y\}$ (why?) and the simplest non-trivial possibility is $\{x, y\}=1$. We then obtain

$$
\begin{aligned}
& \dot{x}=\{x, H\}=-\{H, x\}=-\left(\{x, x\} \frac{\partial H}{\partial x}+\{y, x\} \frac{\partial H}{\partial y}\right)=\frac{\partial H}{\partial y} \\
& \dot{y}=\{y, H\}=-\{H, y\}=-\left(\{x, y\} \frac{\partial H}{\partial x}+\{y, y\} \frac{\partial H}{\partial y}\right)=-\frac{\partial H}{\partial x}
\end{aligned}
$$

and the Poisson bracket reads

$$
\{f, g\}=\frac{\partial f}{\partial x}\{x, g\}+\frac{\partial f}{\partial y}\{y, g\}=\frac{\partial f}{\partial x} \frac{\partial g}{\partial y}-\frac{\partial f}{\partial y} \frac{\partial g}{\partial x} .
$$

Exercise 3.8. Show that there is exactly one Poisson bracket on $\mathbb{R}^{2 n}$ with

$$
\begin{aligned}
& \left\{x_{i}, x_{j}\right\}=0 \\
& \left\{y_{i}, y_{j}\right\}=0 \\
& \left\{x_{i}, y_{j}\right\}=\delta_{i j}, \quad i, j=1, \ldots, n .
\end{aligned}
$$

Such co-ordinates are called canonical co-ordinates. Write down the equations of motion to find out why.

Exercise 3.9. Define a Poisson bracket on $\mathbb{R}^{2 n+\ell}$ with co-ordinates $x_{1}, \ldots, x_{n}$, $y_{1}, \ldots, y_{n}, z_{1}, \ldots, z_{\ell}$ by means of

$$
\begin{aligned}
& \left\{x_{i}, x_{j}\right\}=0, \quad\left\{y_{i}, y_{j}\right\}=0, \quad\left\{x_{i}, y_{j}\right\}=\delta_{i j} \\
& \left\{x_{i}, z_{j}\right\}=0, \quad\left\{y_{i}, z_{j}\right\}=0, \quad\left\{z_{i}, z_{j}\right\}=0 .
\end{aligned}
$$

Show that

$$
\bigwedge_{f, g \in C^{\infty}\left(\mathbb{R}^{2 n+\ell}\right)}\{f, g\}=\sum_{i=1}^{n} \frac{\partial f}{\partial x_{i}} \frac{\partial g}{\partial y_{i}}-\frac{\partial f}{\partial y_{i}} \frac{\partial g}{\partial x_{i}} .
$$

Exercise 3.10. Determine all Poisson structures on $\mathbb{R}^{2}$.

Exercise 3.11. Let $\mathcal{P}$ be a Poisson manifold. Show that the Lie bracket of two Hamiltonian vector fields $X_{H}$ and $X_{K}$, with $H, K \in C^{\infty}(\mathcal{P})$, satisfies

$$
\left[X_{H}, X_{K}\right]=-X_{\{H, K\}}
$$

\subsection{Examples on $S^{2}$}

Using the co-ordinates $(x, y, z)$ on $\mathbb{R}^{3}$ allows us to work globally on $S^{2}=$ $\left\{x^{2}+y^{2}+z^{2}=1\right\}$. Exercise 3.1 suggests to work with 


$$
\{x, y\}=z, \quad\{y, z\}=x, \quad\{z, x\}=y,
$$

as does the $S O(3)$-invariance of $S^{2} \subseteq \mathbb{R}^{3}$. Since

$$
\begin{aligned}
& \{\cos q, p\}=\left\{\frac{x}{\sqrt{1-z^{2}}}, z\right\}=\frac{-y}{\sqrt{1-z^{2}}}=-\sin q \\
& \{\sin q, p\}=\left\{\frac{y}{\sqrt{1-z^{2}}}, z\right\}=\frac{x}{\sqrt{1-z^{2}}}=\cos q
\end{aligned}
$$

cylindrical co-ordinates are canonical co-ordinats for this Poisson structure, i.e. satisfy $\{q, p\}=1$.

For the Hamiltonian function $H(x, y, z)=z$ (measuring the height on the sphere) the equations of motion are

$$
\begin{aligned}
& \dot{x}=\{x, H\}=-y \\
& \dot{y}=\{y, H\}=x \\
& \dot{z}=\{z, H\}=0
\end{aligned}
$$

and easily solved ; the flow is given by

$$
\varphi_{t}(x, y, z)=(x \cos t-y \sin t, x \sin t+y \cos t, z)
$$

and the orbits coincide with the intersections $S^{2} \cap\{H=h\}$ of the phase space with the energy level sets, see Fig. ?.

Definition 3.4. Let $\mathcal{P}$ be a Poisson manifold. A function $f \in C^{\infty}(\mathcal{P})$ is called a Casimir function if

$$
\bigwedge_{g \in C^{\infty}(\mathcal{P})}\{f, g\}=0
$$

For the above Poisson structure $f(x, y, z)=x^{2}+y^{2}+z^{2}$ is a Casimir function and therefore invariant under every Hamiltonian flow on $\mathbb{R}^{3} \supseteq S^{2}$. Every Hamiltonian function $H \in C^{\infty}\left(\mathbb{R}^{3}\right)$ defines a whole family of Hamiltonian systems on the spheres $S_{r}^{2}=f^{-1}\left(r^{2}\right)$ - or a single Hamiltonian system on $\mathbb{R}^{3}$. Note that the gradient of the Casimir is given by

$$
\nabla f(x, y, z)=2\left(\begin{array}{l}
x \\
y \\
z
\end{array}\right)
$$

whence the tangent plane at the point $(x, y, z) \in S_{r}^{2} \subseteq \mathbb{R}^{3}$ is embedded in the Euclidean space $\mathbb{R}^{3}$ as

$$
T_{(x, y, z)} S_{r}^{2}=\left(\begin{array}{l}
x \\
y \\
z
\end{array}\right)+\left(\begin{array}{l}
x \\
y \\
z
\end{array}\right)^{\perp}
$$

and consists of all possible vectors $X_{H}(x, y, z)$ where $H$ runs through all of $C^{\infty}\left(\mathbb{R}^{3}\right)$. 
Example 3.5. Consider on $S^{2}$ (or, in fact, on $\mathbb{R}^{3}$ ) the Hamiltonian function

$$
H(x, y, z)=\frac{a x^{2}}{2}+\frac{b y^{2}}{2}+\frac{c z^{2}}{2}
$$

where $0<a \leq b \leq c$ are three real parameters. (This models part of the dynamics of a free rigid body with a fixed point, subject only to its own inertia.) The equations of motion

$$
\begin{aligned}
& \dot{x}=\{x, H\}=(b-c) y z \\
& \dot{y}=\{y, H\}=(c-a) x z \\
& \dot{z}=\{z, H\}=(a-b) x y
\end{aligned}
$$

are non-linear but can still be explicitly solved, using elliptic functions. Since the orbits coincide with the intersections $S^{2} \cap\{H=h\}$ of the phase space with the energy level sets one can alternatively obtain the phase portrait intersecting the sphere $S^{2} \subseteq \mathbb{R}^{3}$ with the ellipsoid $\{H=h\}$ see Fig. ?.

Exercise 3.12. Analyse the dynamics defined by (3.4) on $S^{2}$ in the limiting cases $a \rightarrow b$ and $b \rightarrow c$.

Exercise 3.13. Study the dynamics of "Colombo's top" on $S^{2}$ with Poisson structure (3.3), the 2-parameter family with Hamiltonian functions

$$
H_{\lambda, \mu}(x, y, z)=-\frac{1}{2}(z-\lambda)^{2}+\mu y
$$

(This models the (averaged) influence of the sun on the motion of a planet revolving around its axis.) Hints: try to answer the following questions. How do the phase portraits look like? (How can they be obtained without lengthy computations?) What is the minimal number of equilibria? How many are there at most? Which bifurcation do you expect where the number of equilibria changes? Can you confirm this numerically? How do the bifurcation curves in the $(\lambda, \mu)$-plane look like?

Exercise 3.14. Let $K \in C^{\infty}\left(\mathbb{R}^{3}\right)$. Show that the triple product

$$
\{f, g\}_{K}:=\langle\nabla f \times \nabla g \mid \nabla K\rangle
$$

(combining the inner product $\langle\mid\rangle$ and the cross product .. $\times$..) defines a Poisson bracket $\{,\}_{K}$ on $\mathbb{R}^{3}$. Let furthermore $H \in C^{\infty}\left(\mathbb{R}^{3}\right)$. How are the flows of $X_{H}$ on $\left(\mathbb{R}^{3},\{,\}_{K}\right)$ and $X_{K}$ on $\left(\mathbb{R}^{3},\{,\}_{H}\right)$ related to each other? 



\section{Theoretical background}

The Poisson bracket is our basic structure, so a few general statements should be made.

Exercise 4.1. Let $\mathcal{P}$ be a Poisson manifold and $F, G \in C^{\infty}(\mathcal{P})$ two constants of motion of the Hamiltonian system defined by $H \in C^{\infty}(\mathcal{P})$, i.e. the flow of $X_{H}$ leaves $F$ and $G$ invariant. Show that the function $\{F, G\} \in C^{\infty}(\mathcal{P})$ is a constant of motion as well.

Exercise 4.2. A Lie algebra is a (real) vector space $\mathfrak{g}$ together with an alternating bilinear form

$$
[,]: \mathfrak{g} \times \mathfrak{g} \longrightarrow \mathfrak{g}
$$

that satisfies the Jacobi identity. Let $v_{1}, \ldots, v_{\ell}$ be a basis of $\mathfrak{g}$. The real numbers $C_{i j}^{k}$ in the representation

$$
\left[v_{i}, v_{j}\right]=\sum_{k=1}^{\ell} C_{i j}^{k} v_{k}
$$

are called the structure constants of the Lie algebra. Show that

$$
\{f, g\}:=\sum_{i, j, k=1}^{\ell} C_{i j}^{k} z_{k} \frac{\partial f}{\partial z_{i}} \frac{\partial g}{\partial z_{j}}
$$

defines a Poisson bracket on $\mathbb{R}^{\ell}$. How do Hamilton's equations look like?

Exercise 4.3. Let $\mathcal{P}$ be a Poisson manifold and $H \in C^{\infty}(\mathbb{R} \times \mathcal{P})$ be a timedependent Hamiltonian function. Rewrite the time-dependent Hamiltonian system defined by $H$ as an autonomous Hamiltonian system on $\mathcal{P} \times \mathbb{R}^{2}$ : construct a Poisson structure on $\mathcal{P} \times \mathbb{R}^{2}$ and define a suitable Hamiltonian function $\widetilde{H} \in C^{\infty}\left(\mathcal{P} \times \mathbb{R}^{2}\right)$. 


\subsection{The structure matrix}

As for all structures in mathematics, the mappings that preserve the structure are (at least) as important as the structure itself.

Definition 4.1. Let $\mathcal{P}_{1}$ and $\mathcal{P}_{2}$ be two Poisson spaces. A mapping $\varphi: \mathcal{P}_{1} \longrightarrow \mathcal{P}_{2}$ is called a Poisson mapping if

$$
\bigwedge_{f, g \in C^{\infty}\left(\mathcal{P}_{2}\right)}\{f \circ \varphi, g \circ \varphi\}_{1}=\{f, g\}_{2} \circ \varphi .
$$

In particular, a Poisson mapping turns canonical co-ordinates into canonical co-ordinates.

Exercise 4.4. Let $H \in C^{\infty}\left(\mathcal{P}_{2}\right)$ be a Hamiltonian function. Show that a Poisson mapping $\varphi: \mathcal{P}_{1} \longrightarrow \mathcal{P}_{2}$ maps the trajectories of the Hamiltonian system defined by the Hamiltonian function $H \circ \varphi$ on $\mathcal{P}_{1}$ into the trajectories of the Hamiltonian system $X_{H}$ on $\mathcal{P}_{2}$.

If a Poisson mapping $\varphi$ is a diffeomorphism, then the inverse $\varphi^{-1}: \mathcal{P}_{2} \longrightarrow \mathcal{P}_{1}$ is also a Poisson mapping and all questions about Hamiltonian systems that one can ask in $\mathcal{P}_{1}$ may be studied in $\mathcal{P}_{2}$.

Theorem 4.2. Let $\varphi$ denote the global flow of a Hamiltonian vector field $X_{H}$ defined by $H \in C^{\infty}(\mathcal{P})$. Then the time-t-mapping $\varphi_{t}: \mathcal{P} \longrightarrow \mathcal{P}$ is a Poisson mapping.

Proof. Given $f, g \in C^{\infty}(\mathcal{P})$, we aim to thow that

$$
\bigwedge_{t \in \mathbb{R}}\left\{f \circ \varphi_{t}, g \circ \varphi_{t}\right\} \circ \varphi_{-t}=\{f, g\} .
$$

Differentiating the left hand side of (4.1) with respect to time yields the expression

$$
\left\{X_{H}\left(f \circ \varphi_{t}\right), g \circ \varphi_{t}\right\}+\left\{f \circ \varphi_{t}, X_{H}\left(g \circ \varphi_{t}\right)\right\}-X_{H}\left(\left\{f \circ \varphi_{t}, g \circ \varphi_{t}\right\}\right)
$$

transformed by $\varphi_{-t}$, where we used trilinearity and

$$
X_{H}(f) \circ \varphi_{t_{0}}=\left.\frac{\mathrm{d}}{\mathrm{d} t}\left(f \circ \varphi_{t_{0}} \circ \varphi_{t-t_{0}}\right)\right|_{t=t_{0}}=X_{H}\left(f \circ \varphi_{t_{0}}\right) .
$$

The Jacobi identity in $F=f \circ \varphi_{t}, G=g \circ \varphi_{t}$ and $H$ implies that the expression (4.2) vanishes. Since (4.1) is true for the "initial condition" $\varphi_{0}=\mathrm{id}$ we conclude that this equation remains true for all times.

In local co-ordinates $\left(z_{1}, \ldots, z_{m}\right)$ the Poisson structure is completely determined by the Poisson brackets $\left\{z_{i}, z_{j}\right\}$ between the co-ordinate functions. Indeed, iterating the expansion (3.1) yields 


$$
\{f, g\}=\sum_{i=1}^{m} \frac{\partial f}{\partial z_{i}}\left\{z_{i}, g\right\}=\sum_{i, j=1}^{m} \frac{\partial f}{\partial z_{i}}\left\{z_{i}, z_{j}\right\} \frac{\partial g}{\partial z_{j}}
$$

whence prescribing the structure matrix

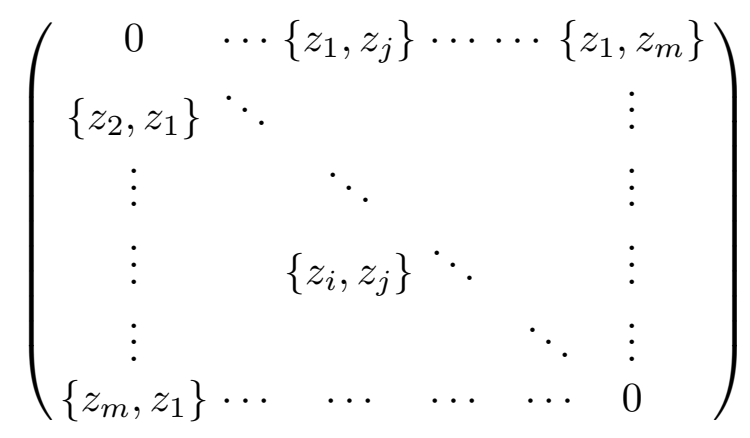

is equivalent to defining a Poisson structure (provided that the Jacobi identity is fulfilled). The structure matrix is anti-symmetric and hence the rank is even in every point.

Definition 4.3. We say that a Hamiltonian system on a Poisson manifold $\mathcal{P}$ has $n$ degrees of freedom if $2 n$ is the maximal rank of the structure matrix $\left(\left\{z_{i}, z_{j}\right\}\right)_{i j}$ on $\mathcal{P}$.

The subset of $\mathcal{P}$ on which the Poisson structure has maximal rank is open (and in non-pathological cases also dense). The origin of $\mathbb{R}^{3}$ with the Poisson brackets (3.3) is an example of a point where the rank of the Poisson structure is not maximal.

Exercise 4.5. Let $(\mathcal{P},\{\}$,$) be a Poisson manifold of even dimension. Show$ that the structure matrix is invertible for every point in a suitable chart around $a \in \mathcal{P}$ if and only if for every function $f \in C^{\infty}(\mathcal{P})$ for which $a$ is not a critical point there is another function $g \in C^{\infty}(\mathcal{P})$ such that $\{f, g\}(a) \neq 0$.

On the open subset where the Poisson structure has maximal rank we can obtain a particularly simple form for the structure matrix. The proof of this result uses Frobenius' theorem.

The linear independent set $\left\{Y_{1}, \ldots, Y_{\ell}\right\}$ of vector fields on the $m$-dimensional manifold $\mathcal{P}$ is said to be involutive if there are coefficient functions $h_{i j}^{k} \in C^{\infty}(\mathcal{P})$ with

$$
\left[Y_{i}, Y_{j}\right]=\sum_{k=1}^{\ell} h_{i j}^{k} \cdot Y_{k}
$$

More generally a distribution $E_{z}<T_{z} \mathcal{P}$ of $\ell$-dimensional subspaces of the tangent spaces of $\mathcal{P}$ is called involutive if for any two vector fields $X, Y$ with values $X_{z}, Y_{z} \in E_{z}$ also $[X, Y]_{z} \in E_{z}$ for all $z$. 
Theorem 4.4. (Frobenius). Let $\left(E_{z}\right)_{z \in \mathcal{P}}$ be an involutive $\ell$-dimensional distribution on the $m$-dimensional manifold $\mathcal{P}$. Then around each point $z \in \mathcal{P}$ there are local co-ordinates $\left\{z_{1}, \ldots, z_{m}\right\}$ such that the first $\ell$ partial derivatives $\frac{\partial}{\partial z_{i}}$, $i=1, \ldots, \ell \operatorname{span} E_{z}$.

The resulting co-ordinate systems $\left\{z_{1}, \ldots, z_{\ell}\right\}$ match up to form $\ell$-dimensional manifolds $M$ for which the tangent spaces $T_{z} M$ at each point $z \in M$ coincide with $E_{z}$. These immersed submanifolds form the leaves of a foliation and a distribution is called integrable if it consists of the tangent spaces of a foliation. Thus Frobenius' theorem states that every involutive distribution is integrable (the converse is trivially true).

Exercise 4.6. Prove Frobenius' theorem for $\ell=1$.

Thus, for a single vector field $Y$ without equilibria there exist local coordinates such that $Y=\frac{\partial}{\partial z_{1}}$, locally the vector field can be straightened out.

Exercise 4.7. Let $\left\{Y_{1}, \ldots, Y_{\ell}\right\}$ be an involutive set of linear independent vector fields. Is it possible to locally straighten out all $\ell$ vector fields simultaneously?

Theorem 4.5. (Darboux). Let $\mathcal{P}$ be a Poisson manifold and assume that the structure matrix has maximal rank $2 n$ in $a \in \mathcal{P}$. Then there are local coordinates $x_{1}, \ldots, x_{n}, y_{1}, \ldots, y_{n}, z_{1}, \ldots, z_{\ell}($ with $2 n+\ell=m:=\operatorname{dim} \mathcal{P})$ around $a$ in which the structure matrix is given by $(3.2 \mathrm{a})$.

Proof. We work by induction on the number $n$ of degrees of freedom. For $n=0$ the structure matrix vanishes in every co-ordinate system and there is nothing to prove (as $\ell=m$ ).

When $n \geq 1$ there exists a Hamiltonian $H \in C^{\infty}(\mathcal{P})$ with $X_{H}(a) \neq 0$. Take $x:=t$ for the time along the trajectories of $X_{H}$ and put $y:=H$. Then

$$
1=\frac{\mathrm{d} t}{\mathrm{~d} t}=\dot{t}=\{t, H\}=\{x, y\}=\{y,-x\}
$$

and we can also interprete $y$ as the time along the (locally defined) Hamiltonian vector field $X_{-x}$. For $f, g \in C^{\infty}(\mathcal{P})$ the Poisson bracket $\{f, g\} \in C^{\infty}(\mathcal{P})$ does not depend on $x$ :

$$
\begin{aligned}
\frac{\partial\{f, g\}}{\partial x} & =\frac{\mathrm{d}\{f, g\}}{\mathrm{d} t}=\{\{f, g\}, H\}=\{\{f, g\}, y\} \\
& =\{f,\{g, y\}\}+\{\{f, y\}, g\}=0
\end{aligned}
$$

and similarly $\{f, g\}$ does not depend on $y$. Therefore, the structure matix is of the form 


$$
\left(\begin{array}{rrr}
0 & 1 & 0 \\
-1 & 0 & 0 \\
0 & 0 & *
\end{array}\right)
$$

where $*$ contains Poisson brackets from co-ordinates in

$$
Z:=\left\{f \in C^{\infty}(\mathcal{P}) \mid\{x, f\}=0=\{y, f\}\right\} .
$$

To show that the functions in $Z$ can indeed be used to complete $x$ and $y$ to a local co-ordinate system we can use Frobenius' theorem as $\left\{X_{y}, X_{x}\right\}$ is involutive. Hence $*$ is itself the structure matrix of a Poisson bracket, of rank $2(n-1)$, so applying the induction hypothesis yields the desired result.

We have proven a bit more than stated in the theorem, the condition that the rank of the Poisson structure be maximal in $a$ was nowhere used.

Corollary 4.6. (Weinstein). Under the conditions of Theorem 4.5, if the rank $2 n$ of the structure matrix in $a$ is not maximal, then there are local co-ordinates $x_{1}, \ldots, x_{n}, y_{1}, \ldots, y_{n}, z_{1}, \ldots, z_{\ell}$ around $a$ in which the structure matrix is given by

$$
\begin{array}{lll}
\left\{x_{i}, x_{j}\right\}=0, & \left\{y_{i}, y_{j}\right\}=0, \quad\left\{x_{i}, y_{j}\right\}=\delta_{i j} \\
\left\{x_{i}, z_{j}\right\}=0, & \left\{y_{i}, z_{j}\right\}=0, & \left\{z_{i}, z_{j}\right\}=S(z)
\end{array}
$$

with $S(0)=0$.

Exercise 3.11 shows that the space of all Hamiltonian vector fields is involutive, so the theorem of Frobenius provides integral manifolds - the integral manifold through a point $a \in \mathcal{P}$ consists of all points $z \in \mathcal{P}$ that can be reached from $a$ by a concatenation of trajectories of (various) Hamiltonian vector fields. The dimension of the integral manifold through $a$ is equal to the rank of the Poisson structure in $a$, one speaks of symplectic leaves. Where this dimension drops one has to use a generalization of Frobenius' theorem due to Stefan and Sussmann.

\subsection{The theorem of Liouville}

Hamiltonian systems conserve the Hamiltonian function, i.e. (for simple mechanical systems) the energy. However, the reason that one often speaks of 'conservative systems' is the conservation of phase space volume.

Theorem 4.7. (Liouville). Hamiltonian systems are volume-preserving.

Proof. The time- - -mapping $\varphi_{t}$ of the Hamiltonian vector field $X_{H}$ transforms a region $\Omega$ into $\varphi_{t}(\Omega)$ with volume 


$$
\begin{aligned}
\int_{\varphi_{t}(\Omega)} & \mathrm{d} x_{1} \cdots \mathrm{d} x_{n} \mathrm{~d} y_{1} \cdots \mathrm{d} y_{n} \mathrm{~d} z_{1} \cdots \mathrm{d} z_{\ell} \\
= & \int_{\Omega} \operatorname{det}\left(D \varphi_{t}\right) \mathrm{d} x_{1} \cdots \mathrm{d} x_{n} \mathrm{~d} y_{1} \cdots \mathrm{d} y_{n} \mathrm{~d} z_{1} \cdots \mathrm{d} z_{\ell}
\end{aligned}
$$

where we work in co-ordinates provided by Darboux's theorem. How does this quantity change in time, say at $t=0$ ? Expanding

$$
D \varphi_{t}=D \varphi_{0}+\left.t \frac{\mathrm{d}}{\mathrm{d} t} D \varphi_{t}\right|_{t=0}+\mathcal{O}\left(t^{2}\right)=\mathrm{id}+t A+\mathcal{O}\left(t^{2}\right)
$$

and using

$$
\operatorname{det}(\lambda \mathrm{id}-A)=\lambda^{n}-\operatorname{trace}(A) \lambda^{n-1}+\ldots \pm \operatorname{det} A
$$

in the form

$$
\operatorname{det}(\mathrm{id}+t A)=1+t \operatorname{trace}(A)+\mathcal{O}\left(t^{2}\right)
$$

we obtain

$$
\begin{aligned}
\left.\frac{\mathrm{d}}{\mathrm{d} t} \operatorname{det}\left(D \varphi_{t}\right)\right|_{t=0} & =\operatorname{trace}(A)=\operatorname{trace}\left(D X_{H}\right) \\
& =\sum_{i=1}^{n} \frac{\partial}{\partial x_{i}} \frac{\partial H}{\partial y_{i}}-\frac{\partial}{\partial y_{i}} \frac{\partial H}{\partial x_{i}}+\sum_{k=1}^{\ell} \frac{\partial}{\partial z_{k}} 0=0
\end{aligned}
$$

For a vector field $Y$ one calls $\operatorname{div}(Y)=\operatorname{trace}(D Y)$ the divergence of $Y$.

Corollary 4.8. The flow of a divergence-free vector field is volume-preserving.

The phase space volume is contracted near an attractor of a dynamical system.

Exercise 4.8. Show that in a Hamiltonian system no asymptotically stable equilibria or periodic solutions occur.

Exercise 4.9. Show that a planar dynamical system, defined on $\mathbb{R}^{2}$, is Hamiltonian if and only if it is area-preserving.

Exercise 4.10. Let $H \in C^{\infty}\left(\mathbb{R}^{2}\right)$ be a Hamiltonian function and assume that for $h_{0} \in \mathbb{R}$ the motion in the level curve $H^{-1}\left(h_{0}\right)$ is periodic. Show that for energy values $h$ near $h_{0}$ the motion is periodic as well. Let $A(h)$ denote the area enclosed by the level $H^{-1}(h)$, while $T(h)$ denotes the period of oscillation in this level set. Show that $T=\frac{\mathrm{d} A}{\mathrm{~d} h}$.

The proof of the next corollary consists of "a walk in the park" covered by (fresh) snow: however small my shoes are, if I walk long enough through the park then I end up stepping on my own trail. 
Corollary 4.9. (Poincaré's recurrence theorem). If $\Omega \subseteq \mathcal{P}$ has finite non-zero volume and is invariant under the flow, then every neighbourhood $U$ of every point $a \in \Omega$ intersects a trajectory that returns to $U$.

Thus, after dropping milk into a cup of coffee, one just has to wait and eventually the fluids are separated again (and the coffee is cold). The solution to this counter-intuitive riddle is that the recurrence time is easily longer than the age of the universe.

Exercise 4.11. Give an explicit proof of Corollary 4.9.

While the volume of $\mathbb{R}^{m}$ is infinite, so one would have to find an appropriate invariant subset $\Omega$, the energy level sets are often compact. The Lebesgue measure of a co-dimension-1 manifold like $\{H=h\}$ vanishes, but it is possible to define an induced volume on $\{H=h\}$ and then one can apply Poincaré's recurrence theorem.

Obviously the notion of "phase space volume" has to be clarified a bit. In $\mathbb{R}^{m}$ with Poisson structure (3.2) it coincides with the Lebesgue measure. For the more general situation that the phase space is a manifold the phase space volume should be a measure that in Darboux co-ordinates coincides with the Lebesgue measure, i.e. may be patched together from such local expressions (one always can resort to dividing a subset $\Omega \subseteq \mathcal{P}$ into small subsets $\Omega_{i}$ that fit into the charts of an atlas and then compute the volume of $\Omega$ as the sum of the volumes of the $\Omega_{i}$ ).

A global definition of the volume on a manifold uses differentiable forms, $\Theta \in \Gamma\left(\mathcal{P}, \Lambda^{m} T^{*} \mathcal{P}\right)$ is called a volume form in much the same way that the Jacobi determinant describes the infinitesimal change of volume in (4.3). The situation is particularly simple if the structure matrix of the Poisson structure on $\mathcal{P}$ is invertible. Then $m=2 n$ and the Poisson structure can be defined in terms of a so-called symplectic form $\omega \in \Gamma\left(\mathcal{P}, \Lambda^{2} T^{*} \mathcal{P}\right)$, locally given by $\omega=\sum \mathrm{d} x_{i} \wedge \mathrm{d} y_{i}$, whence $\Theta=\omega \wedge \ldots \wedge \omega$ is the (Liouville) volume form on $\mathcal{P}$. See Appendix A for a few more details and the modern literature on mechanics for much more details on symplectic forms. 



\section{The planar Kepler system}

Kepler $^{1}$ described the planetary motion in terms of three laws.

1. The planetary orbit is an ellipse with the sun at one of the two focal points.

2. The planetary speed varies in such a way that the line joining the sun and the planet sweeps out equal areas of the ellipse in equal times.

3. The square of the planet's period is proportional to the cube of the semimajor axis of its orbit.

As Newton ${ }^{2}$ showed, these laws can be derived from a gravitational force proportional to the inverse square of the distance. The potential of this force is proportional to the inverse of the distance.

Denoting the vector from the sun to the planet by $x \in \mathbb{R}^{3}$ and their relative speed by $v \in \mathbb{R}^{3}$ the motion is confined to the plane spanned by $x$ and $v$, the plane perpendicular to the angular momentum vector $x \times v \in \mathbb{R}^{3}$. We may choose this plane to be $\mathbb{R}^{2}$. Furthermore placing the centre of the two masses at $0 \in \mathbb{R}^{2}$ we can reduce the problem to that of the motion in a central force field.

Exercise 5.1. Fill in details to explicitly reduce the two body problem to (two times) the problem of the motion of one body in a central force field.

For $U:] 0, \infty[\longrightarrow \mathbb{R}$ we define the Hamiltonian function

$$
H(x, y)=\frac{y_{1}^{2}+y_{2}^{2}}{2}+U\left(x_{1}^{2}+x_{2}^{2}\right)
$$

on $\mathbb{R}^{2 \backslash\{0\}} \times \mathbb{R}^{2}$ (with canonical Poisson bracket), in case of the Kepler system

$$
U(z)=-\frac{\gamma}{\sqrt{z}}
$$

\footnotetext{
${ }^{1}$ Astronomia Nova (1609) and Harmonice Mundi (1619).

2 Philosophiae Naturalis Principia Mathematica (1687).
} 
We first concentrate on properties that hold true for all central force field motions and then consider the special case of the gravitational potential (5.1b).

Exercise 5.2. Draw a potential $V(x)$ with $V(0)=+\infty$ and analyse the Hamiltonian system defined by $H(x, y)=\frac{1}{2} y^{2}+V(x)$ on $] 0, \infty[\times \mathbb{R}$.

Exercise 5.3. Determine the phase portraits of the family of Hamiltonian systems defined on $] 0, \infty\left[\times \mathbb{R}\right.$ by $H(q, p)=\frac{p^{2}}{2}-\frac{1}{q}+\frac{\mu^{2}}{2 q^{2}}$ for a significant choice of values of the parameter $\mu \in \mathbb{R}$.

\subsection{Central force fields}

The potential energy in the Hamiltonian (5.1) is invariant under rotations in the $x$-plane and the kinetic energy is invariant under rotations in the $y$-plane. The combined group action

$$
\begin{aligned}
\psi: S^{1} \times \mathbb{R}^{4} & \left.\longrightarrow\left(\begin{array}{l}
x_{1} \\
x_{2} \\
y_{1} \\
y_{2}
\end{array}\right)\right)
\end{aligned}
$$

furthermore preserves the Poisson structure, i.e. the mapping $\psi_{\rho}$ on the phase space is for every $\rho \in S^{1}$ a Poisson mapping. Consequently, the Hamiltonian vector field is equivariant with respect to $\psi$ and the flow $\varphi$ commutes with this group action $-\varphi_{t} \circ \psi_{\rho}=\psi_{\rho} \circ \varphi_{t}$ for all $(t, \rho) \in \mathbb{R} \times S^{1}$. Our aim is to use this to reduce the system to one degree of freedom.

Exercise 5.4. Let $\psi: \mathcal{P} \longrightarrow \mathcal{P}$ be a Poisson mapping and $H \in C^{\infty}(\mathcal{P})$ be a Hamiltonian function satisfying $H \circ \psi=H$. Explain why $\psi$ is a symmetry of the Hamiltonian system $X_{H}$.

Declaring two points to be equivalent if there is a rotation $\psi_{\rho}$ that turns one into the other we are led to the equivalence classes

$$
\left[\begin{array}{l}
x \\
y
\end{array}\right]=\left\{\left(\begin{array}{l}
\xi \\
\eta
\end{array}\right) \in \mathbb{R}^{4} \mid \bigvee_{\rho \in S^{1}}\left(\begin{array}{l}
\xi \\
\eta
\end{array}\right)=\psi_{\rho}\left(\begin{array}{l}
x \\
y
\end{array}\right)\right\}
$$

and the quotient space

$$
\mathbb{R}^{4} / S^{1}=\left\{\left[\begin{array}{l}
x \\
y
\end{array}\right] \mid\left(\begin{array}{l}
x \\
y
\end{array}\right) \in \mathbb{R}^{4}\right\} .
$$

To be able to define the equations of motion on the abstract quotient space we want to find a concrete realization. The key to this are the invariant functions 


$$
\begin{aligned}
\tau_{1} & =\frac{x_{1}^{2}+x_{2}^{2}}{2} \\
\tau_{2} & =\frac{y_{1}^{2}+y_{2}^{2}}{2} \\
\tau_{3} & =x_{1} y_{1}+x_{2} y_{2} \\
\tau_{4} & =x_{1} y_{2}-x_{2} y_{1}
\end{aligned}
$$

(the fourth quantity is the component of $x$ with respect to the vector $y^{\perp}$ perpendicular to $y$ ).

Theorem 5.1. The ring $\left(C^{\infty}\left(\mathbb{R}^{4}\right)\right)^{S^{1}}$ of $S^{1}$-invariant functions on $\mathbb{R}^{4}$ is generated by $\tau_{1}, \ldots, \tau_{4}$, i.e. every $S^{1}$-invariant function $f \in C^{\infty}\left(\mathbb{R}^{4}\right)$ can be written

$$
f(x, y)=F(\tau(x, y))
$$

as a function $F$ in the variables $\tau_{1}, \ldots, \tau_{4}$.

Proof. In complex co-ordinates $u=x_{1}+\mathrm{i} x_{2}, v=y_{1}+\mathrm{i} y_{2}$ the $S^{1}$-action reads

$$
\psi_{\rho}(u, v)=\left(\mathrm{e}^{\mathrm{i} \rho} u, \mathrm{e}^{\mathrm{i} \rho} v\right)
$$

and leaves the monomial $u^{k} \bar{u}^{l} v^{m} \bar{v}^{n}$ invariant if and only if $l-k=m-n$. In this case we can write the monomial in the form

$$
u^{k} \bar{u}^{l} v^{m} \bar{v}^{n}=(u \bar{u})^{k}(v \bar{v})^{n}(u \bar{v})^{l-k}=\tau_{1}^{k} \tau_{2}^{n}\left(\tau_{3}-\mathrm{i} \tau_{4}\right)^{l-k}
$$

proving the theorem for the ring $\left(\mathbb{R}\left[x_{1}, x_{2}, y_{1}, y_{2}\right]\right)^{S^{1}}$ of $S^{1}$-symmetric polynomials. The extension from polynomials to all smooth functions works for every compact group, see [25, 23].

In the same way that $x_{1}, x_{2}, y_{1}, y_{2}$ are (global) variables on $\mathbb{R}^{4}$ in that every function $f \in C^{\infty}\left(\mathbb{R}^{4}\right)$ can be written as $f \circ\left(x_{1}, x_{2}, y_{1}, y_{2}\right)$, the four $S^{1}$ symmetric functions $\tau_{1}, \tau_{2}, \tau_{3}, \tau_{4}$ serve as global variables on $\mathbb{R}^{4} / S^{1}$, which are restricted by the relations

$$
\tau_{1} \geq 0, \quad \tau_{2} \geq 0 \text { and } \frac{\tau_{3}^{2}+\tau_{4}^{2}}{2}=2 \tau_{1} \tau_{2} .
$$

This last equality is the syzygy of the Hilbert mapping

$$
\tau: \mathbb{R}^{4} \longrightarrow \mathbb{R}^{4}
$$

and we have realized $\mathbb{R}^{4} / S^{1} \cong \operatorname{im} \tau$ as a subset of $\mathbb{R}^{4}$ defined by the relations (5.4). Since the defining constraints (5.4) combine polynomial equations with polynomial inequalities one speaks of a semi-algebraic variety. The Poisson bracket relations yield the structure matrix

$$
\left(\begin{array}{cccc}
0 & \tau_{3} & 2 \tau_{1} & 0 \\
-\tau_{3} & 0 & -2 \tau_{2} & 0 \\
-2 \tau_{1} & 2 \tau_{2} & 0 & 0 \\
0 & 0 & 0 & 0
\end{array}\right)
$$


whence in

$$
\{f, g\}(\tau)=\sum_{i, j=1}^{3} \frac{\partial f}{\partial \tau_{i}}(\tau)\left\{\tau_{i}, \tau_{j}\right\} \frac{\partial g}{\partial \tau_{j}}(\tau)
$$

the summation only has to go to 3 instead of 4 . The rank of this matrix equals 2 , so we have reduced from two to one degree of freedom. The Casimir function $\tau_{4}=x_{1} y_{2}-x_{2} y_{1}$ is the angular momentum which is therefore invariant under the flow of every $S^{1}$-invariant Hamiltonian.

Exercise 5.5. Show that Kepler's second law holds true for all central force fields.

Using $\tau_{4}$ as Hamiltonian function (before the reduction) yields the vector field

$$
X_{\tau_{4}}:\left\{\begin{array}{l}
\dot{x}_{1}=\left\{x_{1}, \tau_{4}\right\}=-x_{2} \\
\dot{x}_{2}=x_{1} \\
\dot{y}_{1}=-y_{2} \\
\dot{y}_{2}=y_{1}
\end{array}\right.
$$

for which the flow is given by (5.2). In particular, the angle $\rho$ can be interpreted as the time variable for this vector field, whence $\left\{\rho, \tau_{4}\right\}=1$.

Mimicking the proof of Darboux's Theorem 4.5 one can obtain canonical co-ordinates $\left(q, \rho, p, \tau_{4}\right)$ with $q=\sqrt{2 \tau_{1}}$, i.e. $(q, \rho)$ are polar co-ordinates on the configuration space $\mathbb{R}^{2 \backslash\{0\}}$. Invariance under (5.2) makes the Hamiltonian in these variables independent of $\rho$ whence $\dot{\tau}_{4}=0$ again follows. A (canonical) co-ordinate is called cyclic if it does not enter the Hamiltonian (whence its conjugate co-ordinate is a conserved quantity). In exercises 5.3 and 5.8 the (planar) Kepler problem is treated in terms of $\left(q, \rho, p, \tau_{4}\right)$.

It follows from Darboux's Theorem 4.5 that the Poisson structure (5.5) admits local co-ordinates $(X, Y, Z, S)$ with $\{X, Y\}=1$ for which $Z$ and $S$ are local Casimir functions. One can take $Z=\tau_{4}$, and also $S$ turns out to be globally defined, given by

$$
S(\tau)=\frac{\tau_{3}^{2}+\tau_{4}^{2}}{2}-2 \tau_{1} \tau_{2}
$$

whence the syzygy $S \equiv 0$ is preserved for every Hamiltonian system. The zero level set $S^{-1}(0)=\{S=0\} \subseteq \mathbb{R}^{4}$ is a double cone, with vertex at the origin $\tau=0$. At this point the rank of the Poisson structure drops from 2 to 0 , whence it is an equilibrium for all Hamiltonian systems.

Exercise 5.6. How is the above reduction of the symmetry (5.2) related to the group $S O(2,1)$ of linear mappings on $\mathbb{R}^{3}$ that preserve the quadratic form $z_{1}^{2}+z_{2}^{2}-z_{3}^{2} ?$

Exercise 5.7. Reduce the $S^{1}$-action 


$$
\begin{aligned}
\phi: S^{1} \times \mathbb{R}^{4} & \left.\longrightarrow\left(\begin{array}{l}
x_{1} \\
x_{2} \\
y_{1} \\
y_{2}
\end{array}\right)\right)
\end{aligned}
$$

on $\mathbb{R}^{4}$ and compute the Poisson bracket on $\mathbb{R}^{4} / S^{1}$ induced by the canonical Poisson structure on $\mathbb{R}^{4}$.

The Hamiltonian function (5.1) induces a Hamiltonian system on the realization

$$
\left(\mathbb{R}^{2 \backslash\{0\}} \times \mathbb{R}^{2}\right) / S^{1}=\left\{\tau \in \mathbb{R}^{4} \mid \tau_{1}>0, \tau_{2} \geq 0, S(\tau)=0\right\}
$$

of the quotient (5.3) with Hamiltonian function

$$
H(\tau)=\tau_{2}+U\left(2 \tau_{1}\right)
$$

that extends to $\left\{\tau \in \mathbb{R}^{4} \mid \tau_{1} \neq 0\right\}$. Since furthermore $\tau_{4}=\mu$ is conserved, the reduced dynamics takes place on the embedded surfaces

$$
\mathcal{P}_{\mu}=\left\{\left(\tau_{1}, \tau_{2}, \tau_{3}\right) \in \mathbb{R}^{3} \mid \tau_{1}>0, \tau_{2} \geq 0, S_{\mu}\left(\tau_{1}, \tau_{2}, \tau_{3}\right)=0\right\}
$$

where

$$
S_{\mu}\left(\tau_{1}, \tau_{2}, \tau_{3}\right)=\frac{\tau_{3}^{2}}{2}-2 \tau_{1} \tau_{2}+\frac{\mu}{2}
$$

The conic sections $S_{\mu}=0$ defined by this quadratic function are two-sheeted hyperboloids for $\mu \neq 0$ and a double cone (now in $\mathbb{R}^{3}$ ) for $\mu=0$. The inequality $\tau_{2} \geq 0$ selects one sheet of the hyperboloid, and since $\tau_{1}>0$ the remaining part of the cone is lacking the $\tau_{2}$-axis. In particular, all surfaces $\mathcal{P}_{\mu}$ are diffeomorphic to the plane $\mathbb{R}^{2}$, see Fig. ?.

Exercise 5.8. Put $q:=\sqrt{2 \tau_{1}}$ and compute the conjugate co-ordinate $p$, i.e. the function $p=p(\tau)$ with $\{q, p\}=1$ and $\{\rho, p\}=0$. Show that the Hamiltonian function (5.1) turns into

$$
H_{\mu}(q, p)=\frac{p^{2}}{2}-\frac{1}{q}+\frac{\mu^{2}}{2 q^{2}}
$$

on $] 0, \infty[\times \mathbb{R}$.

The phase portraits of the one-degree-of-freedom problem on $\mathcal{P}_{\mu}$ with Hamiltonian function $H_{\mu}\left(\tau_{1}, \tau_{2}, \tau_{3}\right)=H\left(\tau_{1}, \tau_{2}, \tau_{3}, \mu\right)$ defined by (5.8) are given by the intersections of the energy level sets $\left\{H_{\mu}=h\right\} \subseteq \mathbb{R}^{3}$ with the phase space $\mathcal{P}_{\mu} \subseteq \mathbb{R}^{3}$. The latter is a surface of revolution (around the axis $\tau_{1}=\tau_{2}$ ) and the former is a 'cylinder', a direct product $B_{h} \times \mathbb{R}$ on the basis 


$$
B_{h}=\left\{\left(\tau_{1}, \tau_{2}\right) \in \mathbb{R}^{2} \mid \tau_{2}=h-U\left(2 \tau_{1}\right)\right\}
$$

in $\mathbb{R}^{2}$. Thus, we can obtain the orbits from the relative position of the two curves $\mathcal{P}_{\mu} \cap\left\{\tau_{3}=0\right\}$ and $\left\{H_{\mu}\left(\tau_{1}, \tau_{2}, 0\right)=h\right\}=B_{h}$ within $\mathbb{R}^{2}$. For $\mu=0$ the former is the positive $\tau_{1}$-axis $\left\{\tau_{1}>0, \tau_{2}=0\right\}$ and for $\mu \neq 0$ it is the hyperbola

$$
\tau_{2}=\frac{\mu^{2}}{4 \tau_{1}} .
$$

Fixing $\mu$ and varying the energy value $h$ results in "moving the basis $B_{h}$ up or down" and yields Fig. ?. From this one easily obtains the phase portraits.

The reduced flows are organized by the (relative) equilibria, where the two surfaces touch each other. This can only happen in the $\left(\tau_{1}, \tau_{2}\right)$-plane as the surface of revolution $\mathcal{P}_{\mu}$ has nowhere else tangent planes that contain the $\tau_{3}-$ axis. Equilibria are therefore given by the points where the two planar curves $\mathcal{P}_{\mu} \cap\left\{\tau_{3}=0\right\}$ and $B_{h}$ touch. For $\mu=0$ the strictly monotonous functions

$$
\tau_{2}=h+\frac{\gamma}{\sqrt{2 \tau_{1}}}
$$

can intersect the $\tau_{1}$-axis only transversely, whence there are no equilibria in this case. For $\mu \neq 0$ the hyperbola (5.10) touches the graph of (5.11) if and only if the difference function

$$
V_{\mu}\left(\tau_{1}\right)=U\left(2 \tau_{1}\right)+\frac{\mu^{2}}{4 \tau_{1}}-h
$$

has a double zero. The equation $V_{\mu}\left(\tau_{1}\right)=0$ can always be fulfilled by adjusting the value $h$ of the energy accordingly. The remaining equation

$$
V_{\mu}^{\prime}\left(\tau_{1}\right)=2 U^{\prime}\left(2 \tau_{1}\right)-\frac{\mu^{2}}{4 \tau_{1}^{2}} \stackrel{!}{=} 0
$$

is for each $\mu$ an equation in $\tau_{1}$ with solution

$$
\tau_{1}=\frac{\mu^{4}}{2 \gamma^{2}}
$$

whence

$$
\tau_{2}=\frac{\gamma^{2}}{2 \mu^{2}} \quad \text { and } \quad h=\frac{-\gamma^{2}}{2 \mu^{2}} .
$$

Thus, as $h$ passes through this value, the motion changes from "no motion possible" to periodic orbits around this equilibrium. For $h=0$ one calls (5.12) the effective potential; in exercise 5.8 the co-ordinates $\left(q, \rho, p, \tau_{4}\right)$ allow to reduce to the 1 -dimensional particle moving in the potential $V_{\mu}$ analysed in exercise 5.3. 
Another qualitative change occurs as $h$ passes through 0 . The right turning point of the periodic orbits (i.e. the right intersection point of the two planar curves) diverges to infinity as $h \nearrow 0$. Since

$$
\dot{\tau}_{1}=\left\{\tau_{1}, H_{\mu}\right\}=\tau_{3}=\sqrt{4 \tau_{1} \tau_{2}-\mu^{2}} \leq A \tau_{1}+B
$$

the solutions with energy $h \geq 0$ are defined for all times, the flow is complete for all $\mu \neq 0$. For $\mu=0$ the energy level curves $\left\{H_{0}=h \geq 0\right\}$ on the lower and upper part of the cone become separated and form two orbits. As $\tau_{1} \rightarrow 0$ the $\tau_{2}$-component accelerates according to

$$
\dot{\tau}_{2}=\left\{\tau_{2}, H_{0}\right\}=-\frac{\partial H_{0}}{\partial \tau_{1}} \tau_{3}=\frac{\gamma \tau_{3}}{2\left(2 \tau_{1}\right)^{\frac{3}{2}}}=\frac{\gamma\left(2 \tau_{2}\right)^{\frac{3}{2}}}{2 \tau_{3}^{2}}
$$

and reaches infinity in finite time (the curve on the upper part of the cone took finite time to leave infinity). The orbits on $\mathcal{P}_{0}$ with negative energy combine this behaviour and collide for both positive and negative finite time with the singularity at $\tau_{1}=0$.

Exercise 5.9. Two particles with mass $m_{1}, m_{2}$ attract each other with gravitational force proportional to the inverse square of the distance. In the initial position, they are at rest and their distance is $d$. When do they meet?

The reduced orbits with angular momentum $\mu=0$ correspond to motion along straight lines in the configuration space $\mathbb{R}^{s \backslash\{0\}}$. Reducing the symmetry (5.2) identified all these directions, whence reconstructing the full flow in two degrees of freedom merely yields a whole circle $S^{1}$ of identical trajectories for each energy value. If the kinetic energy is smaller than the potential energy, then the trajectory collides with the origin in finite time, both in the future and in the past. For $h \geq 0$ the trajectory can escape (in positive or negative time, respectively), when $h=0$ the escape velocity at infinity vanishes.

For angular momenta $\mu \neq 0$ the circle $S^{1}$ attached to each point of the hyperboloid $\mathcal{P}_{\mu}$ does carry dynamics. Thus, from the equilibrium $\tau=$ $\left(\tau_{1}, \tau_{2}, 0, \mu\right)$ given by $(5.13)$ we reconstruct a periodic orbit. In configuration space $\mathbb{R}^{2 \backslash\{0\}}$ this phase space orbit projects to a circle

$$
x_{1}^{2}+x_{2}^{2}=2 \tau_{1}=\frac{\mu^{4}}{\gamma^{2}}
$$

on which the equation of motion derives from (5.9) as

$$
\dot{\rho}=\frac{\partial H_{\mu}}{\partial \tau_{4}}=\frac{\tau_{4}}{2 \tau_{1}}=\frac{\gamma^{2}}{\mu^{3}} .
$$

In particular, the equation

$$
\left(\frac{2 \pi \mu^{3}}{\gamma^{2}}\right)^{2}=\frac{4 \pi^{2}}{\gamma}\left(\frac{\mu^{2}}{\gamma}\right)^{3}
$$

relates period and radius as predicted by Kepler's third law. 
Exercise 5.10. Derive the $\frac{1}{d}$-form of the gravitational potential from Kepler's third law.

For periodic orbits on $\mathcal{P}_{\mu}$ the two intersection points of the hyperbola (5.10) and the graph of (5.11) yield an inner and an outer radius in configuration space $\mathbb{R}^{2 \backslash\{0\}}$ between which (the projection of) the reconstructed trajectory is captured. Passing on phase space to co-ordinates $\left(x_{1}, x_{2}, \tau_{3}, \tau_{4}\right)$, with inverse given by

$$
\begin{aligned}
& y_{1}=\frac{x_{1} \tau_{3}-x_{2} \tau_{4}}{x_{1}^{2}+x_{2}^{2}} \\
& y_{2}=\frac{x_{2} \tau_{3}+x_{1} \tau_{4}}{x_{1}^{2}+x_{2}^{2}}
\end{aligned}
$$

allows us to fix the fourth co-ordinate $\tau_{4}$ and identify the torus

$$
\left\{\left(x_{1}, x_{2}, \tau_{3}, \tau_{4}\right) \in \mathbb{R}^{4} \mid \tau_{4}=\mu, H=h\right\}
$$

in Fig. ?. The motion on this invariant manifold is conditionally periodic, superposing the periodic motion on $\mathcal{P}_{\mu}$ with the periodic motion in $\rho$ along the attached circles. In the limit $h \rightarrow \frac{-\gamma^{2}}{2 \mu^{2}}$ the torus shrinks down to the periodic orbit in the $x$-plane $\left\{\tau_{3}=0\right\}$. As $h$ passes to positive values, the outer radius goes to infinity (and beyond) whence the torus turns into a cylinder. The flow on (5.14) commutes with the $S^{1}$-action (5.2) which turns trajectories into trajectories, only rotating in $x$ and leaving the co-ordinates $\tau_{3}$ and $\tau_{4}$ fixed.

Exercise 5.11. Analyse the behaviour of an orbit in a (planar) central force field that has total energy equal to the effective potential energy at a local maximum.

Exercise 5.12. What do the results on planar central force fields imply for the free particle?

\subsection{The eccentricity vector}

The energy-momentum mapping

$$
\begin{aligned}
& \mathcal{E M}: \mathbb{R}^{4} \longrightarrow \quad \mathbb{R}^{2} \\
& (x, y) \mapsto\left(\tau_{4}(x, y), H(x, y)\right)
\end{aligned}
$$

allows to collect the information on the global dynamics that we obtained so far, see Fig. ?. Shown are the bifurcation values of $\mathcal{E M}$, the remaining open parts consist of regular values. 
For values $(\mu, h) \in \mathbb{R}^{2}$ satisfying $h<\frac{-\gamma^{2}}{2 \mu^{2}}$ the inverse image $\mathcal{E M}^{-1}(\mu, h)$ is empty. It is only by convention that these are called regular values; with this definition the statement of Sard's Lemma is simply that the regular values of a smooth mapping form an open and dense set of full measure - the complement (the critical values) has Lebesgue measure zero. The regular values in

$$
\left\{(\mu, h) \in \mathbb{R}^{2} \mid \mu \neq 0, \frac{-\gamma^{2}}{2 \mu^{2}}<h<0\right\}
$$

have invariant tori as inverse images, on which the periodic orbit $\mathcal{P}_{\mu} \cap H_{\mu}^{-1}(h)$ is superposed with the periodic motion in the $\rho$-variable that has been suppressed when reducing the symmetry (5.2).

For a general central force field this conditionally periodic motion alternates between quasi-periodic - the trajectory lies dense on the torus - and periodic - the trajectory returns exactly to its initial state after having superposed the two periodic motions for a finite number of periods. Furthermore, it will in general depend on the initial condition, and thus on the values $\mu$ of $\tau_{4}$ and $h$ of $H$, which of these two alternatives applies. A torus $\mathcal{E M}^{-1}(\mu, h)$ that consists of periodic orbits is also called resonant, since the frequencies $\omega_{1}, \omega_{2}$ satisfy a resonance relation

$$
k_{1} \omega_{1}+k_{2} \omega_{2}=0
$$

with an integer vector $k \in \mathbb{Z}^{2 \backslash\{0\}}$ that one may choose to have relative prime components. The periodic motion then consists of $k_{2}$ periods on the reduced phase space superposed with $k_{1}$ full rotations about the origin $x=0$.

According to Kepler's first law, all invariant 2-tori have to be resonant, with $k_{1}=k_{2}=1$ in (5.16). While following an ellipse in configuration space $\mathbb{R}^{2 \backslash\{0\}}$, the trajectory moves along the "lower" part of the torus when approching the origin and along the "upper" part when elongating (indeed, $\dot{\tau}_{1}=\tau_{3}$ ), see Fig. ?. Such a uniform resonance, valid for all invariant tori, hints to the existence of an additional conserved quantity (and hence to an extra symmetry).

Theorem 5.2. (Noether). Let $\left(\psi_{s}\right)_{s \in \mathbb{R}}$ be the flow of a Hamiltonian vector field defined by $F \in C^{\infty}(\mathcal{P})$ for which every $\psi_{s}: \mathcal{P} \longrightarrow \mathcal{P}$ is a symmetry of the Hamiltonian system $X_{H}$. Then $F$ is a conserved quantity (or first integral) of $X_{H}$.

Exercise 5.13. Prove Noether's theorem. Is the converse true as well?

The vector

$$
\frac{\tau_{4}}{\gamma} y^{\perp}-\frac{1}{\sqrt{2 \tau_{1}}} x=\frac{\tau_{4}}{\gamma}\left(\begin{array}{c}
y_{2} \\
-y_{1}
\end{array}\right)-\frac{1}{\sqrt{2 \tau_{1}}}\left(\begin{array}{c}
x_{1} \\
x_{2}
\end{array}\right)
$$

is called the eccentricity vector of the planar Kepler system. The first component is conserved since 


$$
\begin{aligned}
\frac{\mathrm{d}}{\mathrm{d} t}\left[\frac{\tau_{4} y_{2}}{\gamma}-\frac{x_{1}}{\sqrt{2 \tau_{1}}}\right] & =\frac{\tau_{4} \dot{y}_{2}}{\gamma}-\frac{\dot{x}_{1}}{\sqrt{2 \tau_{1}}}+\frac{x_{1} \dot{\tau}_{1}}{\left(2 \tau_{1}\right)^{\frac{3}{2}}} \\
& =\frac{\tau_{4}}{\gamma} \frac{-\gamma x_{2}}{\left(x_{1}^{2}+x_{2}^{2}\right)^{\frac{3}{2}}}-\frac{y_{1}}{\sqrt{2 \tau_{1}}}+\frac{x_{1} \tau_{3}}{\left(2 \tau_{1}\right)^{\frac{3}{2}}} \\
& =\frac{-\tau_{4} x_{2}-2 \tau_{1} y_{1}+x_{1} \tau_{3}}{\left(x_{1}^{2}+x_{2}^{2}\right)^{\frac{3}{2}}} \\
& =0 .
\end{aligned}
$$

The second component differs from the first only by interchanging $x_{1} \leftrightarrow x_{2}$ and $y_{1} \leftrightarrow y_{2}$, the minus sign in $y^{\perp}$ is compensated by the resulting minus sign in $\tau_{4}=x_{1} y_{2}-x_{2} y_{1}$. Thus, the second component is conserved as well.

Four independent constants of motion would make every point an equilibrium. For the length of (5.17) we obtain

$$
\begin{aligned}
\left\|\frac{\tau_{4}}{\gamma} y^{\perp}-\frac{1}{\sqrt{2 \tau_{1}}} x\right\|^{2} & =\frac{\tau_{4}^{2} \cdot 2 \tau_{2}}{\gamma}-2 \frac{\tau_{4}}{\gamma \sqrt{2 \tau_{1}}}\left\langle y^{\perp} \mid x\right\rangle+1 \\
& =\frac{2 \tau_{4}^{2}}{\gamma^{2}}\left(\tau_{2}-\frac{\gamma}{\sqrt{2 \tau_{1}}}\right)+1 \\
& =\frac{2 \tau_{4}^{2} H}{\gamma^{2}}+1 .
\end{aligned}
$$

The direction of the eccentricity vector (5.17) cannot be invariant under the rotation (5.2), so we have found a third independent constant of motion. As

$$
x\left\|\frac{\tau_{4}}{\gamma} y^{\perp}-\frac{1}{\sqrt{2 \tau_{1}}} x \Longleftrightarrow x\right\| y^{\perp} \Longleftrightarrow x \perp y
$$

this direction points to one of the two turning points of the ellipse where $\tau_{1}$ is extremal (and $\tau_{3}$ vanishes).

To confirm that the projection of the orbit to the configuration plane $\mathbb{R}^{2 \backslash\{0\}}$ is indeed an ellipse, with focal points at the origin and at

$$
f=\frac{1}{H}\left(\frac{\tau_{4}}{\gamma} y^{\perp}-\frac{1}{\sqrt{2 \tau_{1}}} x\right)
$$

we consider the sum of the distance to the two focal points. Since the time derivative

$$
\begin{aligned}
\frac{\mathrm{d}}{\mathrm{d} t}[\|x\|+\|x-f\|] & =\frac{\mathrm{d}}{\mathrm{d} t}\left[\sqrt{2 \tau_{1}}+\sqrt{2 \tau_{1}-\frac{2}{H}\left(\tau_{4}^{2}-\sqrt{2 \tau_{1}}\right)+\frac{2 \tau_{4}^{2} H+1}{H^{2}}}\right] \\
& =\frac{\tau_{3}}{\sqrt{2 \tau_{1}}}\left(1+\frac{\sqrt{2 \tau_{1}}+H^{-1}}{\sqrt{2 \tau_{1}+2 H^{-1} \sqrt{2 \tau_{1}}+H^{-2}}}\right)
\end{aligned}
$$

vanishes (check!), this sum remains constant along the orbit which is therefore an ellipse. 
Exercise 5.14. Show that the eccentricity vector points away from the centre of the ellipse.

For $h \rightarrow 0$ the second focus point wanders off to infinity and the ellipses limit a parabola, turning into a hyperbola for positive energies. Thus, the cylinders $\mathcal{E M}^{-1}(\mu, h), h>0$, are foliated (and even fibrated) by curves that make less than a full rotation in the $x$-plane when passing from $\tau_{3}=-\infty$ to $\tau_{3}=+\infty$.

Exercise 5.15. The correction of the gravitational law in general relativity leads in first order to the Hamiltonian function

$$
H(x, y)=\frac{y_{1}^{2}+y_{2}^{2}}{2 m}-\frac{\gamma}{\sqrt{x_{1}^{2}+x_{2}^{2}}}+\frac{\varepsilon \tau_{4}^{2}}{x_{1}^{2}+x_{2}^{2}} \quad, \quad 0<\varepsilon=\frac{\gamma}{m^{2} c^{2}} \ll 1 .
$$

What happens to "Kepler's ellipses" under the influence of this perturbation? Is this also true for the other conic sections?

The bifurcation set of the energy-momentum mapping contains next to the $\mu$-axis also the $h$-axis, i.e. the values for which the orbit can disappear into the origin. Another special property of the Kepler system, compared to other central force fields, is that this collision singularity can be regularized. We concentrate on the bounded orbits.

Exercise 5.16. Consider the energy level set $\left\{H=-\frac{1}{2 \lambda^{2}}\right\}$ and apply the co-ordinate transformation $(x, y) \mapsto(q, p)$ defined by

$$
\begin{aligned}
x_{1} & =\frac{\lambda}{2}\left(q_{1}^{2}-q_{2}^{2}\right), & y_{1} & =\frac{\lambda}{4} \frac{q_{1} p_{1}-q_{2} p_{2}}{q_{1}^{2}+q_{2}^{2}} \\
x_{2} & =\lambda q_{1} q_{2}, & y_{2} & =\frac{\lambda}{4} \frac{q_{1} p_{2}+q_{2} p_{1}}{q_{1}^{2}+q_{2}^{2}}
\end{aligned}
$$

together with the scaling

$$
\frac{\mathrm{d} s}{\mathrm{~d} t}=\frac{1}{2 \lambda \sqrt{q_{1}^{2}+q_{2}^{2}}}
$$

of time. Show that this regularizes the singularity at the origin. Can you give an interpretation of the part $q \mapsto x$ of the transformation?

The third part of the bifurcation set of the energy-momentum mapping parametrises the circular orbits with minimal effective potential energy. When considering the fibration of the invariant tori $\mathcal{E M}^{-1}(\mu, h), h<0$ by ellipses the circular orbits "fit in" to make $\{H<0\}$ an $S^{1}$-bundle, i.e. every periodic orbit of the Kepler system has a neighbourhood that is diffeomorphic to $S^{1} \times \mathbb{R}^{3}$. Let us compute the base space $\{H=h\} / S^{1}$ of this bundle for fixed negative energy. Writing the expression for the length of the eccentricity vector as 


$$
\frac{\left\|\frac{\tau_{4} y^{\perp}}{\gamma}-\frac{x}{\sqrt{2 \tau_{1}}}\right\|^{2}}{-2 H}+\frac{\tau_{4}^{2}}{\gamma^{2}}=\frac{1}{-2 H}
$$

suggests to work with the invariants

$$
\begin{aligned}
\sigma_{1} & =\frac{\frac{\tau_{4} y_{2}}{\gamma}-\frac{x_{1}}{\sqrt{2 \tau_{1}}}}{\sqrt{-2 H}} \\
\sigma_{2} & =\frac{-\frac{\tau_{4} y_{1}}{\gamma}-\frac{x_{2}}{\sqrt{2 \tau_{1}}}}{\sqrt{-2 H}} \\
\sigma_{3} & =\frac{\tau_{4}}{\gamma} \\
\sigma_{4} & =\frac{1}{\sqrt{-2 H}}
\end{aligned}
$$

which indeed generate the ring of $X_{H}$-invariant functions.

Exercise 5.17. Apply the transformation (5.18) to $\sigma_{1}, \sigma_{2}, \sigma_{3}, \sigma_{4}$ and conclude that every polynomial (smooth) conserved quantity can be written as a polynomial (smooth function) in these variables.

The Poisson bracket relations between these variables yield the structure matrix

$$
\frac{1}{\gamma}\left(\begin{array}{cccc}
0 & \sigma_{3} & -\sigma_{2} & 0 \\
-\sigma_{3} & 0 & \sigma_{1} & 0 \\
\sigma_{2} & -\sigma_{1} & 0 & 0 \\
0 & 0 & 0 & 0
\end{array}\right)
$$

and we recover (3.3). Hence, the two Casimirs are given by $\sigma_{4}$ and $\sigma_{1}^{2}+\sigma_{2}^{2}+\sigma_{3}^{2}$. In particular, the Kepler system admits for negative energy the symmetry group $S O(3)$ and the base space $\{H=h\} / S^{1}$ of the $S^{1}$-bundle can be identified with the $2-$ sphere of radius $\sigma_{4}=\frac{1}{\sqrt{-2 h}}$.

Exercise 5.18. Regularize the singularity at the origin for $h>0$ and show that the Kepler system admits for positive energy the symmetry group $S O(2,1)$.

Adjusting cylindrical co-ordinates on the sphere $\sigma_{1}^{2}+\sigma_{2}^{2}+\sigma_{3}^{2}=\sigma_{4}^{2}$ to $G=2 \pi \gamma \sigma_{3}=2 \pi \tau_{4}$ and conjugate angle $g$ (measuring the direction of the eccentricity vector) we can mimick the proof of Darboux's Theorem 4.5 to obtain canonical co-ordinates $(g, \ell, G, L)$, interpreting $L=2 \pi \sigma_{4}$ as Hamiltonian and $\ell$ as the time along the corresponding flow. In these Delaunay elements the Hamiltonian of the Kepler system is expressed as $H=-\frac{2 \pi^{2}}{L^{2}}$ whence the flow becomes particularly simple as the equations of motion read

$$
\dot{\ell}=\frac{4 \pi^{2}}{L^{3}}, \quad \dot{g}=0, \quad \dot{G}=0, \quad \dot{L}=0
$$


Hence, $(g, \ell)$ are $\mathbb{T}^{2}$-valued variables on the invariant tori $\left\{G=G_{0}, L=L_{0}\right\}$ that are well adapted to the flow. The co-ordinate singularities $G= \pm L$ correspond to the circular orbits.

Exercise 5.19. For a free action $G \times \mathcal{P} \longrightarrow \mathcal{P}$ of a compact Lie group $G$ on the manifold $\mathcal{P}$ the quotient $\mathcal{P} / G$ is again a smooth manifold and the mapping $\tau: \mathcal{P} \longrightarrow \mathcal{P} / G$ (that sends each point $x \in \mathcal{P}$ to the $G$-orbit $\{g(x) \mid g \in G\}$ ) is a submersion (i.e. has in each point a surjective derivative). Let now $\mathcal{P}$ be a Poisson manifold and each $g: \mathcal{P} \longrightarrow \mathcal{P}$ be a Poisson mapping. Show that there is a unique Poisson structure on $\mathcal{P} / G$ that makes $\tau$ a Poisson mapping. 



\section{The spherical pendulum}

The pendulum with configuration space $S^{1}$ moves within a vertical plane. Omitting this constraint, the pendulum is allowed to move on the whole sphere $S^{2} \subseteq \mathbb{R}^{3}$, subject only to the constraint $x_{1}^{2}+x_{2}^{2}+x_{3}^{2}=1$. Differentiating with respect to time yields $x_{1} \dot{x}_{1}+x_{2} \dot{x}_{2}+x_{3} \dot{x}_{3}=0$, i.e. the velocity vector has to be perpendicular to the vector of position if the motion is to preserve the sphere. Defining

$$
\begin{aligned}
& R(x, y)=\frac{x_{1}^{2}+x_{2}^{2}+x_{3}^{2}}{2} \\
& Q(x, y)=x_{1} y_{1}+x_{2} y_{2}+x_{3} y_{3}
\end{aligned}
$$

our phase space is given by

$$
\mathcal{P}=\left\{(x, y) \in \mathbb{R}^{3} \times \mathbb{R}^{3} \mid R(x, y)=\frac{1}{2}, S(x, y)=0\right\} .
$$

The Hamiltonian function is the sum

$$
H(x, y)=\frac{y_{1}^{2}+y_{2}^{2}+y_{3}^{2}}{2}+\gamma x_{3}
$$

of kinetic and potential energy. When working with the canonical Poisson structure $\{$,$\} on \mathbb{R}^{6}$ we find

$$
\begin{aligned}
& \dot{R}=\{R, H\}=Q=0 \\
& \dot{Q}=y_{1}^{2}+y_{2}^{2}+y_{3}^{2}-\gamma x_{3} \not \equiv 0
\end{aligned}
$$

and once the value of $Q$ starts changing and becomes non-zero also the value of $R$ begins to deviate from $\frac{1}{2}$.

Exercise 6.1. Determine the phase portraits of the family of Hamiltonian systems defined on $] 0, \pi\left[\times \mathbb{R}\right.$ by $H(q, p)=\frac{p^{2}}{2}-\cos q+\frac{\mu^{2}}{2 \sin ^{2} q}$ for a significant choice of values of the parameter $\mu \in \mathbb{R}$. 


\subsection{Dirac brackets}

There are two ways to ensure that the trajectories of $X_{H}$ do not leave the phase space $\mathcal{P}$, changing the Hamiltonian and changing the Poisson structure. We pursue the second alternative, this makes $\mathcal{P}$ a Poisson submanifold of $\mathbb{R}^{6}$. To distinguish the new Poisson bracket $\{$,$\} from the canonical Poisson bracket$ we denote the latter by $[$,$] . Our aim is to define \{\}=,[]+,\ldots$ in such a way that the constraining functions $R$ and $Q$ become Casimir functions, whence the restriction of $\{$,$\} to \mathcal{P}$ is again a Poisson bracket. To this end we compute

$$
[R, Q]=x_{1}^{2}+x_{2}^{2}+x_{3}^{2}=2 R
$$

and define the Dirac bracket

$$
\{f, g\}=[f, g]+\frac{1}{2 R}[f, R][Q, g]-\frac{1}{2 R}[f, Q][R, g]
$$

whence $\{f, R\}=0$ and $\{f, Q\}=0$ for all $f \in C^{\infty}\left(\mathbb{R}^{6}\right)$. In particular, the equations $R \equiv \frac{1}{2}$ and $Q \equiv 0$ defining $\mathcal{P}$ are conserved for every Hamiltonian $H \in C^{\infty}\left(\mathbb{R}^{6}\right)$.

Exercise 6.2. Given $R_{1}, \ldots, R_{k} \in C^{\infty}\left(\mathbb{R}^{2 n}\right)$, under what condition is it possible to define a Poisson structure on $\mathbb{R}^{2 n}$ with $n-\left\lceil\frac{k}{2}\right\rceil$ degrees of freedom that makes $R_{1}, \ldots, R_{k}$ Casimir functions ?

The relations

$$
\begin{aligned}
& {\left[x_{i}, R\right]=0} \\
& {\left[y_{i}, R\right]=-x_{i}} \\
& {\left[x_{i}, Q\right]=x_{i}} \\
& {\left[y_{i}, Q\right]=-y_{i}}
\end{aligned}
$$

allow to compute the structure matrix

$$
\frac{1}{2 R}\left(\begin{array}{cccccc}
0 & 0 & 0 & x_{2}^{2}+x_{3}^{2} & -x_{1} x_{2} & -x_{1} x_{3} \\
0 & 0 & 0 & -x_{1} x_{2} & x_{1}^{2}+x_{3}^{2} & -x_{2} x_{3} \\
0 & 0 & 0 & -x_{1} x_{3} & -x_{2} x_{3} & x_{1}^{2}+x_{2}^{2} \\
-x_{2}^{2}-x_{3}^{2} & x_{1} x_{2} & x_{1} x_{3} & 0 & -x_{1} y_{2}+x_{2} y_{1} & x_{3} y_{1}-x_{1} y_{3} \\
x_{1} x_{2} & -x_{1}^{2}-x_{3}^{2} & x_{2} x_{3} & x_{1} y_{2}-x_{2} y_{1} & 0 & -x_{2} y_{3}+x_{3} y_{2} \\
x_{1} x_{3} & x_{2} x_{3} & -x_{1}^{2}-x_{2}^{2}-x_{3} y_{1}+x_{1} y_{3} & x_{2} y_{3}-x_{3} y_{2} & 0
\end{array}\right)
$$

whence the equations of motion

$$
\begin{aligned}
& \dot{x}_{i}=\left\{x_{i}, H\right\}=y_{i}-\sum_{j=1}^{3} \frac{x_{i} x_{j} y_{j}}{2 R}=y_{i}-\frac{Q}{2 R} x_{i} \\
& \dot{y}_{i}=\left\{y_{i}, H\right\}=-\gamma \delta_{i 3}+\frac{\gamma x_{3}-y_{1}^{2}-y_{2}^{2}-y_{3}^{2}}{2 R} x_{i}+\frac{Q}{2 R} y_{i}
\end{aligned}
$$


turn on $\mathcal{P}$ into

$$
\dot{x}=y \quad \text { and } \quad \dot{y}=-\left(\begin{array}{l}
0 \\
0 \\
\gamma
\end{array}\right)+\left(\gamma x_{3}-\left(y_{1}^{2}+y_{2}^{2}+y_{3}^{2}\right)\right) \cdot x
$$

after using $R \equiv \frac{1}{2}$ and $Q \equiv 0$. The number of degrees of freedom is now two, coincidings with the number of directions in which the pendulum can move. The second term in the $\dot{y}$-equation is the constraining force that keeps the pendulum on the configuration space $S^{2}$.

Exercise 6.3. Show that the subset $\left\{x_{2}=y_{2}=0\right\}$ of $\mathcal{P}$ is diffeomorphic to $S^{1} \times \mathbb{R}$. Check that the equations of motion defined by (6.1) on this submanifold describe the (planar) mathematical pendulum.

\subsection{Symmetry reduction}

The kinetic energy $\frac{1}{2}\left(y_{1}^{2}+y_{2}^{2}+y_{3}^{2}\right)$ of the spherical pendulum is invariant under the whole group $S O(3)$ of 3 -dimensional rotations and the potential energy $\gamma x_{3}$ is invariant under rotations about the $x_{3}$-axis. The simultaneous rotation

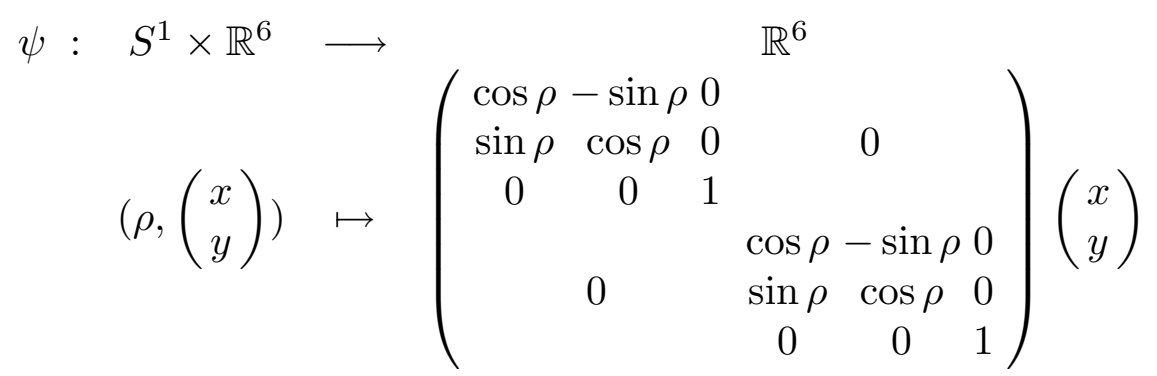

furthermore preserves the Poisson structure whence the Hamiltonian vector field $X_{H}$ is equivariant with respect to the Poisson mapping $\psi_{\rho}$ for every $\rho \in S^{1}$. Again we use invariants to reduce to one degree of freedom and take

$$
\begin{aligned}
\tau_{1} & =\frac{x_{1}^{2}+x_{2}^{2}}{2} \\
\tau_{2} & =\frac{y_{1}^{2}+y_{2}^{2}}{2} \\
\tau_{3} & =x_{1} y_{1}+x_{2} y_{2} \\
\tau_{4} & =x_{1} y_{2}-x_{2} y_{1} \\
\tau_{5} & =x_{3} \\
\tau_{6} & =y_{3}
\end{aligned}
$$

as variables on the reduced phase space. 
Theorem 6.1. The ring $\left(C^{\infty}\left(\mathbb{R}^{6}\right)\right)^{S^{1}}$ of $S^{1}$-invariant functions on $\mathbb{R}^{6}$ is generated by $\tau_{1}, \ldots, \tau_{6}$, i.e. every $S^{1}$-invariant function $f \in C^{\infty}\left(\mathbb{R}^{6}\right)$ can be written as a function in the variables $\tau_{1}, \ldots, \tau_{6}$.

Proof. We can consider a given a polynomial $f \in \mathbb{R}\left[x_{1}, x_{2}, x_{3}, y_{1}, y_{2}, y_{3}\right]$ with real coefficients as a polynomial $f \in \mathbb{R}\left[x_{1}, x_{2}, y_{1}, y_{2}\right]\left[\tau_{5}, \tau_{6}\right]$ in the two variables $x_{3}=\tau_{5}$ and $y_{3}=\tau_{6}$ with coefficients that are itself (real) polynomials $g \in$ $\mathbb{R}\left[x_{1}, x_{2}, y_{1}, y_{2}\right]$ in four variables. For such $g$ invariance under (6.2) is the same as invariance under (5.2) whence the ring of all invariant coefficient polynomials $g$ is generated by $\tau_{1}, \ldots, \tau_{4}$, see Theorem 5.1 . Therefore

$$
\begin{aligned}
\left(\mathbb{R}\left[x_{1}, x_{2}, x_{3}, y_{1}, y_{2}, y_{3}\right]\right)^{S^{1}} & =\left(\mathbb{R}\left[x_{1}, x_{2}, y_{1}, y_{2}\right]\right)^{S^{1}}\left[\tau_{5}, \tau_{6}\right] \\
& =\mathbb{R}\left[\tau_{1}, \tau_{2}, \tau_{3}, \tau_{4}\right]\left[\tau_{5}, \tau_{6}\right] \\
& =\mathbb{R}\left[\tau_{1}, \tau_{2}, \tau_{3}, \tau_{4}, \tau_{5}, \tau_{6}\right] .
\end{aligned}
$$

Again we extend from polynomials to all smooth functions as in [25, 23].

While $\tau_{5}$ and $\tau_{6}$ are free variables, the four $S^{1}$-symmetric functions $\tau_{1}, \tau_{2}, \tau_{3}$ and $\tau_{4}$ are still restricted by the relations

$$
\tau_{1} \geq 0, \quad \tau_{2} \geq 0 \text { and } S(\tau)=0
$$

with (5.6), now defining a function $S: \mathbb{R}^{6} \longrightarrow \mathbb{R}$, entering the syzygy of the Hilbert mapping

$$
\tau: \mathbb{R}^{6} \longrightarrow \mathbb{R}^{6}
$$

We have realized $\mathbb{R}^{6} / S^{1} \cong \operatorname{im} \tau$ as a semi-algebraic subset of $\mathbb{R}^{6}$.

Exercise 6.4. Determine the structure matrix $\left(\left\{\tau_{i}, \tau_{j}\right\}\right)_{i j}$ and show that $2 \tau_{1}+$ $\tau_{5}^{2}, \tau_{3}+\tau_{5} \tau_{6}, 4 \tau_{1} \tau_{2}-\tau_{3}^{2}$ and $\tau_{4}$ define Casimir functions.

The structure matrix has rank 2 (we reduced from two to one degree of freedom) and correspondingly there are four Casimir functions. From the Dirac bracket we inherit

$$
\begin{aligned}
& R(\tau)=\tau_{1}+\frac{\tau_{5}^{2}}{2} \stackrel{!}{=} \frac{1}{2} \\
& Q(\tau)=\tau_{3}+\tau_{5} \tau_{6} \stackrel{!}{=} 0
\end{aligned}
$$

and $S$ is a Casimir function as well. The fourth Casimir function is the variable $\tau_{4}$, the angular momentum about the vertical axis. The values of Casimir functions remain fixed for every Hamiltonian system and we can use this to eliminate the variables

$$
\tau_{1}=\frac{1-\tau_{5}^{2}}{2}, \quad \tau_{3}=-\tau_{5} \tau_{6} \quad \text { and } \quad \tau_{4}=\mu
$$

where $\mu \in \mathbb{R}$ is a parameter with values determined by the initial condition of the spherical pendulum. We transform the remaining variables to 


$$
u:=\tau_{5}=x_{3}, \quad v:=\tau_{6}=y_{3} \quad \text { and } \quad w:=\tau_{2}+\frac{\tau_{6}^{2}}{2}=\frac{y_{1}^{2}+y_{2}^{2}+y_{3}^{2}}{2}
$$

which are constrained by the relations

$$
|u| \leq 1, \frac{v^{2}}{2} \leq w \text { and } S_{\mu}(x, y, z)=0
$$

with the remaining Casimir taking the form

$$
S_{\mu}(x, y, z)=\frac{v^{2}}{2}-\left(1-u^{2}\right) w+\frac{\mu^{2}}{2} .
$$

The structure matrix reads

$$
\left(\begin{array}{ccc}
0 & 1-u^{2} & v \\
-1+u^{2} & 0 & -2 u w \\
-v & 2 u w & 0
\end{array}\right)
$$

and defines the Poisson bracket

$$
\{f, g\}=-\left\langle\nabla f \times \nabla g \mid \nabla S_{\mu}\right\rangle
$$

on $\mathbb{R}^{3}$.

Exercise 6.5. Analyse the force free spherical pendulum.

For the reduced phase space

$$
\mathcal{P}_{\mu}=\left\{(u, v, w) \in \mathbb{R}^{3}|| u \mid \leq 1, w \geq 0, S_{\mu}(x, y, z)=0\right\}
$$

the inequality $\frac{1}{2} v^{2} \leq w$ is a consequence of the $S_{\mu}=0$, except when $u=0$, which only can happen when $\mu=0$ and in which case necessarily $v=0$ as well. Thus, even the seemingly weaker inequality $w \geq 0$ is an extra constraint only if $\mu=0$. The Hamiltonian function

$$
H_{\mu}(u, v, w)=w+\gamma u
$$

reduced from (6.1) is independent of $v$ and $\mu$, the energy level sets $\left\{H_{\mu}=h\right\}$ are the planes perpendicular to the vector

$$
\left(\begin{array}{l}
1 \\
0 \\
\gamma
\end{array}\right)
$$

and contain for every energy value $h \in \mathbb{R}$ the $v$-axis. From (6.4) and (6.5) we obtain the equations of motion

$$
\begin{aligned}
\dot{u} & =\left\{u, H_{\mu}\right\}=v \\
\dot{v} & =\left\{v, H_{\mu}\right\}=u^{2}-1-2 \gamma u w \\
\dot{w} & =\left\{w, H_{\mu}\right\}=-\gamma v
\end{aligned}
$$


which are defined on $\mathbb{R}^{3}$ but leave $\mathcal{P}_{\mu}$ invariant, and also conserve energy. Hence, the trajectories of $X_{H_{\mu}}$ are the intersections of $\mathcal{P}_{\mu}$ with the planes $\left\{H_{\mu}=h\right\}$.

In case $\mu \neq 0$ the equation $S_{\mu}=0$ implies that $u \neq \pm 1$ and we can divide this equation by $1-u^{2}$ to obtain the explicit expression

$$
w=w(u, v)=\frac{1}{2} \frac{v^{2}-\mu^{2}}{1-u^{2}} .
$$

This makes $\mathcal{P}$ a graph over the $(u, v)$-space $]-1,1[\times \mathbb{R}$, see Fig. ?. Intersections with planes perpendicular to (6.6) are closed curves that shrink down to a point $(u, v, w)=\left(u^{*}, 0, w^{*}\right)$ where the two surfaces touch. Here the reduced flow has its unique equilibrium. Note that the tangent planes of $\mathcal{P}$ at $\left(u_{*}, v_{*}, w_{*}\right)$ contain the $v$-axis if and only if $v_{*}$ vanishes, whence we can compute $u^{*}$ (and hence $w^{*}=w\left(u^{*}, 0\right)$ and the energy $h^{*}=w^{*}-\gamma u^{*}$ ) equating the derivatives of

$$
\frac{1}{2} \frac{-\mu^{2}}{1-u^{2}}=h-\gamma u
$$

with respect to $u$. This yields

$$
\gamma u^{4}-2 \gamma u^{2}+\mu^{2} u+\gamma=0
$$

and the 4 th order polynomial has a unique root $\left.u^{*} \in\right]-1,1[$.

Exercise 6.6. Find an explicit expression of $u^{*}$ and draw the resulting graph as a function of $\mu$. What are the asymptotic values for $\mu \rightarrow 0$ and for $\mu \rightarrow$ $\pm \infty ?$

For $\mu=0$ we first have to generalize Definition 3.2 to allow for singular points.

Definition 6.2. Let $\mathcal{P} \subseteq \mathbb{R}^{m}$ be a closed subset and $\mathcal{A} \subseteq C(\mathcal{P})$ a Poisson algebra. Then we call $(\mathcal{P},\{\}$,$) a Poisson space.$

For $\mathcal{P}_{\mu}$ we work with the Poisson algebra

$$
\mathcal{A}=\left\{F_{\mu} \in C\left(\mathcal{P}_{\mu}\right) \mid \bigvee_{f \in\left(C^{\infty}(\mathcal{P})\right)^{S^{1}}} F_{\mu(x, y)}(u(x, y), v(x, y), w(x, y))=f(x, y)\right\}
$$

which yields $C^{\infty}\left(\mathcal{P}_{\mu}\right)$ for $\mu \neq 0$. However, when $\mu=0$ the reduced phase space $\mathcal{P}_{0}$ no longer is a smooth manifold. The defining equation

$$
\frac{v^{2}}{2}=\left(1-u^{2}\right) w
$$

has for $v=0$ the solution lines

$$
\begin{aligned}
& (u, v, w)=( \pm 1,0, w) \\
& (u, v, w)=(u, 0,0)
\end{aligned}
$$


with free parameters $w \geq 0$ and $u \in[-1,1]$, respectively. Where $v \neq 0$ we have again $u \neq \pm 1$ and divide by $1-u^{2}$ to obtain the explicit expression

$$
w=w(u, v)=\frac{1}{2} \frac{v^{2}}{1-u^{2}} .
$$

Fixing $u$ makes this a parabola, with minimum attained at (6.11). For a better understanding of the singular points at the intersection of (6.10) and (6.11) we rewrite (6.9) as

$$
(u+1)(u-1) w+\frac{v^{2}}{2}=0
$$

and recognize for $u \approx 1$ as well as for $u \approx-1$ the equation for a double cone, which is in both cases restriced to a cone when taking $|u| \leq 1$ into account.

Theorem 6.3. The singular points $(u, v, w)=( \pm 1,0,0)$ are equilibria for every Hamiltonian system on $\mathcal{P}_{0}$.

Proof. The Poisson bracket vanishes at these points and hence also the Hamiltonian vector field.

Intersecting $\mathcal{P}_{0}$ with the planes perpendicular to (6.6) yields the trajectories of $X_{H_{0}}$ whence these can be constructed from Fig. ?. For $\mu=0$ the angular momentum about the vertical axis vanishes. This confines the motion of the spherical pendulum to the plane generated by the initial position $x$ and the initial momentum $y$. The $S^{1}$-action (6.2) turns such initial conditions into initial conditions for oscillations in a different plane, and reducing the $S^{1}$ symmetry identifies all these planes with each other. Taking $\rho=\pi$ in (6.2) shows that the (planar) mathematical pendulum has a $\mathbb{Z}_{2}$-symmetry.

Exercise 6.7. Reduce the $\mathbb{Z}_{2}$-symmetry of the mathematical pendulum.

Exercise 6.8. Construct for $\mu \neq 0$ a diffeomorphism $\left.\mathcal{P}_{\mu} \longrightarrow\right] 0, \pi[\times \mathbb{R}$ that turns the Hamiltonian system defined by $X_{H_{\mu}}$ into the system considered in exercise 6.1 .

Exercise 6.9. Reformulate Definition 6.2 in such a way that the phase space need not be given as a subset of some $\mathbb{R}^{m}$, still covering all the examples met so far.

\subsection{Reconstruction}

To reconstruct the flow of the spherical pendulum from the reduced phase portraits on $\mathcal{P}_{\mu}$ we have to "undo" the identifications of elements of $\mathcal{P}$ that are turned into each other by a rotation $\psi_{\rho}$ of the $S^{1}$-action (6.2). The two singular points on $\mathcal{P}_{0}$ correspond to the two points of $\mathcal{P}$ where (6.2) has non-trivial isotropy. Indeed, for $x_{1}=x_{2}=y_{1}=y_{2}=0$ the point $(x, y) \in$ 
$\mathcal{P}$ is a fixed point of every $\psi_{\rho}, \rho \in[0,2 \pi]$. Hence, the two points $(x, y)=$ $(0,0, \pm 1,0,0,0)$ are not identified with any other point when reducing (6.2). These are the two equilibria of the spherical pendulum, the stable position "hanging down" and the unstable position "standing up".

Exercise 6.10. Compute the eigenvalues of the linearization of the Hamiltonian vector field in the two equilibria.

For regular points on the reduced phase space the inverse image under the Hilbert mapping (6.3) is a circle parametrised by the angle $\rho$ that measures the simultaneous rotation (6.2) about the vertical axis. For regular equilibria on $\mathcal{P}_{\mu}$ this circle is an invariant manifold and since $\dot{\rho} \neq 0$ we obtain the periodic solutions found by Huygens ${ }^{1}$ - the spherical pendulum rotates about the vertical axis, at a fixed height $x_{3}=u^{*}$ that keeps the gravitational and centrifugal forces in equilibrium.

Exercise 6.11. Compute the frequency of the periodic solution for given $u^{*}$.

Where this rotational motion is superposed with a (relative) periodic orbit of the reduced system the motion becomes conditionally periodic, taking place on an invariant 2-torus. For $\mu \neq 0$ the configuration $x(t) \in S^{2}$ of such a conditionally periodic trajectory $(x(t), y(t))$ remains confined between the two circles of height $x_{3}=u$. and $x_{3}=u$. Here $u$., $u$ are the two solutions of $(6.8)$ with $h=H(x(0), y(0))$ that lie in the interval $[-1,1]$. Both periodic and quasiperiodic trajectories occur, with the orbit closing or spinning densely around the 2-torus, respectively.

For $\mu=0$ the circles attached to each regular point of $\mathcal{P}_{\mu}$ carry no dynamics and all invariant tori $\left\{\tau_{4}=0, H=h\right\}$ are resonant. When $h<\gamma$ the resulting periodic orbits are planar oscillations around the stable equilibrium point and when $h>\gamma$ we recover the rotating motions over the top of the mathematical pendulum. The invariant set $\left\{\tau_{4}=0, H=\gamma\right\}$ can be thought of as a torus with the "inner" $\rho$-fibre pinched to the unstable equilibrium point $(x, y)=(0,0,1,0,0,0)$. The stable and unstable manifolds coincide and form together with the equilibrium the pinched torus.

Exercise 6.12. Analyse the spherical pendulum with potential energy $x_{1}^{2}+x_{2}^{2}$ instead of $\gamma x_{3}$.

The energy-momentum mapping

$$
\mathcal{E M}=\left(\tau_{4}, H\right): \mathcal{P} \longrightarrow \mathbb{R}^{2}
$$

allows to collect the information on the global dynamics. For regular values $(\mu, h) \in \operatorname{im} \mathcal{E M}$ the inverse image $\mathcal{E M}^{-1}(\mu, h)$ is an invariant 2-torus while a critical value with $\mu \neq 0$ has a periodic orbit as inverse image. The inverse

\footnotetext{
${ }^{1}$ De Vi Centrifuga (1659) and Horologium Oscillatorium (1673).
} 
image $\mathcal{E M}^{-1}(0,-\gamma)$ is the stable equilibrium and the inverse image $\mathcal{E M}^{-1}(0, \gamma)$ is the pinched torus.

For critical values $(\mu, h)$ the reduced phase space $\mathcal{P}_{\mu}$ touches the energy plane $\left\{H_{\mu}=h\right\}$ whence the polynomial

$$
P_{\mu, h}(u):=\gamma u^{3}-h u^{2}-\gamma u+h-\frac{\mu^{2}}{2}
$$

has a double root. Note that for $\mu=0$ this implies $u= \pm 1$, while for $\mu \neq 0$ the roots of $P_{\mu, h}$ coincide with the roots of (6.8).

Theorem 6.4. The subset

$$
\Delta:=\left\{(\mu, h) \in \mathbb{R}^{2} \mid P_{\mu, h} \text { has multiple roots in }[-1,1]\right\}
$$

of the discriminant locus admits the parametrisation

$$
\begin{aligned}
h & =\frac{3 \gamma}{2} s-\frac{\gamma}{2 s} \\
\mu & = \pm \sqrt{\gamma} \frac{1-s^{2}}{\sqrt{-s}}
\end{aligned}
$$

with parameter $s \in[-1,0[\cup\{+1\}$.

Proof. Factorise

$$
\begin{aligned}
P_{\mu, h}(u) & =\gamma(u-s)^{2}(u-t) \\
& =\gamma u^{3}-\gamma(2 s+t) u^{2}+\gamma\left(2 s t+s^{2}\right) u-\gamma s^{2} t
\end{aligned}
$$

and compare coefficients, this yields $2 s t+s^{2}=-1$ whence $s \neq 0$ for the double root $s \in[-1,1]$. Substituting

$$
t=-\frac{1+s^{2}}{2 s}
$$

in $h=\gamma(2 s+t)$ and $\frac{1}{2} \mu^{2}-h=\gamma s^{2} t$ yields $(6.13 \mathrm{a})$ and

$$
\mu^{2}=-\frac{\gamma}{s}\left(1-s^{2}\right)^{2}
$$

with negative right hand side for $s \in] 0,1[$.

As shown in Fig. ?, the bifurcation set $\Delta$ of $\mathcal{E M}$ is formed by the two arcs corresponding to the stable periodic orbits, both parametrised by $s \in]-1,0[$, the meeting point $(\mu, h)=(0,-\gamma)$ of these two arcs, corresponding to the stable equilibrium and given by $s=-1$ in (6.13), and the isolated point $(\mu, h)=(0, \gamma)$ corresponding to the pinched torus and given by $s=+1$. 
Exercise 6.13. Compute the hook formed by the two arcs of $\Delta$ where they meet.

Exercise 6.14. To model the influence of a magnetic monopole at the origin on the motion of an electrically charged particle in $\mathbb{R}^{3 \backslash\{0\}}$ one adjusts the canonical Poisson bracket from $\left\{y_{i}, y_{j}\right\}=0$ to

$$
\left\{y_{i}, y_{j}\right\}=\varepsilon_{i j k} \frac{a x_{k}}{|x|^{3}}
$$

where $\varepsilon_{i j k}:=\operatorname{sgn}\left(\begin{array}{l}123 \\ i j k\end{array}\right)$ denotes the alternating Levi-Civita symbol. One speaks of a magnetic spherical pendulum if furthermore the constraints $x_{1}^{2}+x_{2}^{2}+x_{3}^{2}=$ 1 and $x_{1} y_{1}+x_{2} y_{2}+x_{3} y_{3}=0$ are fulfilled. The Hamiltonian function is still given by (6.1).

(i) Determine the corresponding Dirac bracket.

(ii) Reduce the axial $S^{1}$-symmetry.

(iii) Give the phase portraits of the reduced magnetic spherical pendulum.

(iv) Determine the critical values of the energy-momentum mapping.

\subsection{Action angle variables}

A co-ordinate $I$ on $\mathcal{P}$ is called an action if all orbits of the flow of $X_{I}$ are periodic. Where one can determine a transverse section of initial conditions the time parametrisation of the periodic flow allows to define a conjugate angle, i.e. a function $\varphi: \mathcal{P} \longrightarrow \mathbb{T}^{1}$ satisfying $\{\varphi, I\}=1$. One speaks of action angle variables for a system in $n$ degrees of freedom if the Hamiltonian $H=H(I)$ depends only on the actions $I_{1}, \ldots, I_{n}$ and not on the angles $\varphi_{1}, \ldots, \varphi_{n}$, whence the equations of motion read

$$
\begin{gathered}
\dot{\varphi}=D_{I} H(\varphi, I)=: \quad \omega(I) \\
\dot{I}=-D_{\varphi} H(\varphi, I)=0
\end{gathered}
$$

with frequency vector $\omega(I) \in \mathbb{R}^{n}$. The trajectory with initial condition $(\varphi(0), I(0))=\left(\varphi^{0}, I^{0}\right)$ is conditionally periodic and spins densely around the torus $\mathbb{T}^{n} \times\left\{I^{0}\right\}$ if the frequencies do not satisfy a resonance relation

$$
k_{1} \omega_{1}+\ldots+k_{n} \omega_{n}=0
$$

with an integer vector $k \in \mathbb{Z}^{n \backslash\{0\}}$. The Delaunay elements are action angle variables for the Kepler system and action angle variables for the harmonic oscillator were constructed in exercise 2.3.

Exercise 6.15. Show that every Hamiltonian system on $\mathbb{R}^{2}$ that has only periodic orbits admits action angle variables. Hint: use exercise 4.10. 
Let $\mathcal{R}=\mathcal{E M}^{-1}(C)$ be the inverse image of the set $C \subseteq \mathbb{R}^{2}$ of regular values of the energy-momentum mapping of the spherical pendulum. The third component of the angular momentum is an action $I_{1}:=2 \pi \tau_{4}$ with conjugate angle $\varphi_{1}:=\frac{\rho}{2 \pi}$. The reduced motion on $\mathcal{P}_{\mu}$ is periodic, and normalizing the period $T$ to 1 allows to define the second angle $\varphi_{2}$. For the conjugate action $I_{2}$ this amounts to

$$
X_{I_{2}}=T(\mu, h) X_{H}-\nu(\mu, h) X_{I_{1}}
$$

where $\nu(\mu, h)$ is the frequency ratio of the conditionally periodic flow on the invariant torus $\mathcal{E M}^{-1}(\mu, h)$.

Constructing action angle variables in the neighbourhood of each torus yields local diffeomorphisms

$$
(\varphi, I): \mathcal{U} \longrightarrow \mathbb{T}^{2} \times V
$$

with open subsets $\mathcal{U} \subseteq \mathcal{R}$ and $V \subseteq \mathbb{R}^{2}$. These are semi-local variables, while the angles $\varphi \in \mathbb{T}^{2}$ are defined globally on every torus contained in $\mathcal{U}$, the actions $I$ are only locally defined (and also the angles are not globally defined on all of $\mathcal{R}$ ). The local diffeomorphisms (6.15) make $\mathcal{R}$ a 2 -torus bundle, every point has a neighbourhood $\mathcal{U}$ that is "trivial" - looking like a direct product. One speaks of global action angle variables if one can put $\mathcal{U}=\mathcal{R}$ in (6.15), whence the torus bundle itself is trivial.

Theorem 6.5. The spherical pendulum does not admit global action angle variables

Proof. For $h$ near $-\gamma$ the Hamiltonian system admits local co-ordinates $(q, p)$ on $\mathcal{P}$ in which the Hamiltonian function can be developed into a Taylor series

$$
H(q, p)=-\gamma+\sqrt{\gamma} \frac{p_{1}^{2}+p_{2}^{2}+q_{1}^{2}+q_{2}^{2}}{2}+\mathcal{O}(3)
$$

whence the energy level set $H^{-1}(h)$ is diffeomorphic to the 3 -sphere $p_{1}^{2}+p_{2}^{2}+$ $q_{1}^{2}+q_{2}^{2}=1$

When $h>\gamma$ the equation $H=h$ determines for fixed $x_{3} \in[-1,1]$ a $y-$ sphere of radius $2 h-2 \gamma x_{3}$, and intersecting with the tangent plane $\langle x \mid y\rangle=0$ yields a circle. Hence, the energy level set $H^{-1}(h)$ is diffeomorphic to the unit tangent bundle

$$
T_{1} S^{2}=\left\{(x, y) \in \mathbb{R}^{6} \mid\|x\|=1,\langle x \mid y\rangle=0,\|y\|=1\right\} .
$$

Completing the vectors $x$ and $y$ by $x \times y$ yields an orthonormal frame, showing that $T_{1} S^{2} \cong S O(3)$, the group of 3 -dimensional rotations.

Except for the identity every rotation has a unique rotation axis, the eigenspace to the eigenvalue 1 . In this way every point in the closed subset

$$
B=\left\{z \in \mathbb{R}^{3} \mid\|z\| \leq 2 \pi\right\}
$$


represents a rotation, with all points on the boundary $\partial B$ representing the identity. The quotient space $B / \partial B$ is diffeomorphic to $S^{3}$. However, each element of $S O(3)$ corresponds to two points in this quotient space. Indeed, rotating an angle $\psi$ about the vector $z$ defines the same rotation as rotating the angle $2 \pi-\psi$ about the opposite vector $-z$.

Thus, $S^{3}$ is a 2:1 covering of $S O(3)$ and the energy level sets for low and high values of $h$ are not diffeomorphic to each other. As shown in [7] this contradicts the assumption that the 2 -torus bundle $\mathcal{R}$ is trivial.

Depending on the initial condition the quasi-periodic motions of the spherical pendulum favour an interpretation of rotating oscillation or oscillating rotation or rotating rotation. The non-existence of global action angle variables reflects that these points of view cannot be reconciled.

Exercise 6.16. Construct action angle variables for the planar motion in a central force field. 


\section{Coupled oscillators}

Let $z \in \mathcal{P}$ be a regular point of a Poisson manifold $\mathcal{P}$ with even dimension $2 n$, where the rank of the Poisson structure in $z$ assumes the maximal possible value $2 n$. For local questions (around $z$ ) we may work in canonical co-ordinates $x_{1}, \ldots, x_{n}, y_{1}, \ldots, y_{n}$ and thus restrict ourselves to the case $z=0 \in \mathbb{R}^{2 n}$ with canonical Poisson bracket. However, where the co-ordinates $x_{1}, \ldots, \ldots, y_{n}$ become distinguished if $0 \in \mathbb{R}^{2 n}$ is the starting point, the seemingly more general starting point above makes it clear that canonical co-ordinate changes do not lead to a different problem but on the contrary may serve to simplify a given problem.

Exercise 7.1. Assume that the derivative of $H \in C^{\infty}\left(\mathbb{R}^{2 n}\right)$ does not vanish at the origin. Show that locally around the origin there are canonical coordinates $q_{1}, \ldots, q_{n}, p_{1}, \ldots, p_{n}$ in which $H(q, p)=p_{1}$ and solve the resulting equations of motion. Can you give a physical interpretation?

Thus, within a sufficiently small neighbourhood the Hamiltonian flows near all points that are not equilibria look alike, leaving only the case $D H(0)=0$ for further local study. The value $H(0)$ having no dynamical meaning we may substract it from the Hamiltonian and assume that $H(0)=0$ whence the Taylor series

$$
H(z)=\sum_{k=2}^{\infty} D^{k} H(0) \cdot \frac{z^{k}}{k !}
$$

starts at order $k=2$. Truncating the higher order terms we obtain a quadratic Hamiltonian function, i.e. we linearize

$$
\dot{z}=D X_{H}(0) \cdot z
$$

the vector field at the equilibrium. Our aim is to find canonical co-ordinates in which the quadratic part $H_{2}$ of $H$ (and hence $D X_{H}(0)=X_{H_{2}}$ ) looks particularly simple. 
Theorem 7.1. Let $H_{2} \in C^{\infty}\left(\mathbb{R}^{2 n}\right)$ be a quadratic Hamiltonian. Then the eigenvalues of the linear vector field $X_{\mathrm{H}_{2}}$ can be grouped into complex quartets $\pm \Re \pm \mathrm{i} \Im$, purely imaginary pairs $\pm \mathrm{i} \Im$, symmetric real pairs $\pm \Re$ and the eigenvalue 0 with even multiplicity.

Proof. Let the matrix $\Omega \in M_{2 n \times 2 n}(\mathbb{R})$ represent the linear mapping $z \mapsto$ $X_{H_{2}}(z)$ in canonical co-ordinates. Then ${ }^{1}$

$$
\Omega^{T}=I \Omega I
$$

where $I \in M_{2 n \times 2 n}(\mathbb{R})$ is given by

$$
I=\left(\begin{array}{cc}
0 & \mathrm{id}_{n} \\
-\mathrm{id}_{n} & 0
\end{array}\right)
$$

with $\operatorname{id}_{n} \in M_{n \times n}(\mathbb{R})$ denoting the identity matrix. Since $I^{2}=-\mathrm{id}_{2 n}$ the characteristic polynomial $\chi_{\Omega} \in \mathbb{R}[\lambda]$ of $\Omega$ satisfies

$$
\begin{aligned}
\chi_{\Omega}(\lambda) & =\operatorname{det}\left(\lambda \mathrm{id}_{2 n}-\Omega\right)=\operatorname{det}\left(\lambda \mathrm{id}_{2 n}-\Omega^{T}\right) \\
& =\operatorname{det}\left(-\lambda I^{2}-I \Omega I\right) \\
& =\operatorname{det}\left(-\lambda \mathrm{id}_{2 n}-\Omega\right)=\chi_{\Omega}(-\lambda) .
\end{aligned}
$$

Hence, for every eigenvalue $\lambda \in \mathbb{C}$ the complex number $-\lambda$ is an eigenvalue as well, as are $\bar{\lambda}$ and $-\bar{\lambda}$ (because $\chi_{\Omega}$ has real coefficients). The quartet $\pm \lambda$, $\pm \bar{\lambda}$ shrinks to pairs if $\bar{\lambda}=\lambda$ or $\bar{\lambda}=-\lambda$ and if both these equations hold true then $\lambda=0$. Denoting by $k, l, m$ the number of distinct quartets, symmetric real pairs and purely imaginary pairs, respectively, the algebraic multiplicity of the eigenvalue zero is equal to $2 n-4 k-2 l-2 m$.

Two linear Hamiltonian systems with different eigenvalues can not be transformed into each other by means of a Poisson transformation; this differentiable mapping would induce a linear transformation between the linear systems. Preservation of the Poisson structure leads to further signs distinguishing different types.

Exercise 7.2. Classify all linear Hamiltonian systems in two degrees of freedom that have no multiple eigenvalues. Hint: consider the Hessian matrix $D^{2} H_{2}=I^{-1} X_{H_{2}}$.

Multiplying the Hamiltonian by -1 amounts to reversing the time direction and allows to reduce the number of cases, e.g. identifying minima with maxima of the Hamiltonian. However, the relative sign cannot be removed, leaving us for two pairs $\pm \mathrm{i} \alpha_{1}, \pm \mathrm{i} \alpha_{2}$ of imaginary eigenvalues with the definite type

$$
H_{2}(q, p)=\alpha_{1} \frac{p_{1}^{2}+q_{1}^{2}}{2}+\alpha_{2} \frac{p_{2}^{2}+q_{2}^{2}}{2}
$$

${ }^{1}$ The matrix $D H_{2}=I^{-1} \Omega$ is symmetric. 
and the indefinite type

$$
H_{2}(q, p)=\alpha_{1} \frac{p_{1}^{2}+q_{1}^{2}}{2}-\alpha_{2} \frac{p_{2}^{2}+q_{2}^{2}}{2}
$$

of coupled oscillators. The linear flow defined by the superposition of two oscillators depends on number-theoretic properties of $\alpha_{1}, \alpha_{2}>0$. Next to the equilibrium $(q, p)=0$ there are periodic orbits

$$
(q(t), p(t))=\left(q_{1}^{0} \cos \alpha_{1} t+\frac{p_{1}^{0}}{\alpha_{1}} \sin \alpha_{1} t, 0,-\alpha_{1} q_{1}^{0} \sin \alpha_{1} t+p_{1}^{0} \cos \alpha_{1} t, 0\right)
$$

and

$$
(q(t), p(t))=\left(0, q_{2}^{0} \cos \alpha_{2} t+\frac{p_{2}^{0}}{\alpha_{2}} \sin \alpha_{2} t, 0,-\alpha_{2} q_{2}^{0} \sin \alpha_{2} t+p_{2}^{0} \cos \alpha_{2} t\right)
$$

for special choices of the initial conditions. All other trajectories lie on invariant tori

$$
T_{c}=\left\{\frac{1}{2}\left(p_{1}^{2}+q_{1}^{2}\right)=c_{1}, \frac{1}{2}\left(p_{2}^{2}+q_{2}^{2}\right)=c_{2}\right\}
$$

with positive constants $c_{1}, c_{2} \in \mathbb{R}$. If the quotient $\frac{\alpha_{1}}{\alpha_{2}}$ is irrational, then every trajectory on every torus $T_{c}$ lies dense. In the resonant case $\frac{\alpha_{1}}{\alpha_{2}} \in \mathbb{Q}$ we write $\frac{\alpha_{1}}{\alpha_{2}}=\frac{k}{\ell}$ with $k, \ell \in \mathbb{N}$ relative prime. All orbits on $T_{c}$ are periodic, rotating $k$ times in the $\left(q_{1}, p_{1}\right)$-plane and $\ell$ times in the $\left(q_{2}, p_{2}\right)$-plane before closing. Dividing the Hamiltonian by the common multiple $k^{-1} \alpha_{1}=\ell^{-1} \alpha_{2}$ we can achieve the standard form

$$
H_{2}(q, p)=k \frac{p_{1}^{2}+q_{1}^{2}}{2} \pm \ell \frac{p_{2}^{2}+q_{2}^{2}}{2}
$$

of the (linear) $k: \pm \ell$ resonance.

This standard form carries over to the 1:1 resonance, where the definiteness of $D^{2} H_{2}$ prevents the occurrence of a nilpotent part in $X_{H_{2}}$. An example of an equilibrium with linear part in 1:1 resonance is the stable rest position $(x, y)=(0,0,-1,0,0,0)$ of the spherical pendulum. Also the equilibrium $(x, y)=0$ of the central force field with potential $U(x)=\frac{1}{2}\left(x_{1}^{2}+x_{2}^{2}\right)$ is in 1:1 resonance. Note that in both examples the symmetry with respect to an $S^{1}$-action prevents a detuning of the frequencies.

Exercise 7.3. What is the simplest form that the linearization of the unstable rest position $(x, y)=(0,0,1,0,0,0)$ of the spherical pendulum can assume in local co-ordinates of the form (3.2)?

An equilibrium in 1:-1 resonance is called semi-simple if the quadratic part of the Hamiltonian can be brought into the standard form

$$
H_{2}(q, p)=\frac{p_{1}^{2}+q_{1}^{2}}{2}-\frac{p_{2}^{2}+q_{2}^{2}}{2} .
$$

In general the occurrence of a nilpotent part cannot be prevented. 


\subsection{The Krein collision}

Bifurcations of equilibria occur where the eigenvalue configuration is changed to a different type. In one degree of freedom this means that a hyperbolic equilibrium becomes elliptic (or vice versa) as e.g. in the Hamiltonian pitchfork bifurcation. Superposing one of the bifurcation scenarios of Section 2.2 with a saddle $H(q, p)=\frac{1}{2} p^{2}-\frac{1}{2} q^{2}$ yield this bifurcation scenario on a normally hyperbolic invariant submanifold. A saddle generated in the bifurcation yields a saddle-saddle equilibrium in two degrees of freedom, and centres lead to saddle-centre equilibria (with one real pair and one purely imaginary pair of eigenvalues). Superposition with a centre similarly results in equilibria of saddle-centre and centre-centre type.

The four types of equilibria in two degrees of freedom that do not have zero eigenvalues have eigenvalue configurations as depicted in Fig. ?. Passages from and to the saddle-centre involve zero eigenvalues and "diagonal passages" would even impose all eigenvalues to vanish at the bifurcation. Both the saddle-saddle equilibrium and the focus-focus equilibrium are hyperbolic equilibria, with no eigenvalue on the imaginary axis. From a local dynamical viewpoint no bifurcation takes place when passing from one to the other since their flows are topologically conjugate on sufficiently small neighbourhoods. There is a homeomorphism mapping trajectories to trajectories, preserving the time parametrisation. This allows to translate possible local questions from one system to the other and later translating back the answer to that question.

The remaining possibility is the passage from an elliptic equillibrium of centre-centre type to a hyperbolic equilibrium of focus-focus type, with intermediate eigenvalue configuration as given in Fig. ?. Both 1:1 resonant equilibria and 1:-1 resonant equilibria have such a double purely imaginary pair of eigenvalues. However, an equilibrium in 1:1 resonance is a local extremum of the Hamiltonian function whence all small deformations of the system have a nearby equilibrium that is a local extremum as well. Hence the deformed Hamiltonian has a quadratic part of the form (7.1), showing that all small deformations of a 1:1 resonant equilibrium are elliptic.

Thus, a 1-parameter family $H^{\lambda}$ of Hamiltonians with an equilibrium $z_{\lambda} \in \mathbb{R}^{4}$ passing from focus-focus type to centre-centre type has to admit a parameter value $\lambda_{0}$ for which the equilibrium is in 1:-1 resonance.

Exercise 7.4. Use the implicit mapping theorem to change to new coordinates in which $z_{\lambda}=0$ for all parameter values $\lambda \in \mathbb{R}$.

For a better understanding of the situation it is helpful to apply the coordinate transformation

$$
\left(\begin{array}{l}
q_{1} \\
q_{2} \\
p_{1} \\
p_{2}
\end{array}\right) \mapsto\left(\begin{array}{l}
x_{1} \\
x_{2} \\
y_{1} \\
y_{2}
\end{array}\right)=\frac{1}{\sqrt{2}}\left(\begin{array}{cccc}
1 & 0 & 0 & -1 \\
0 & 1 & -1 & 0 \\
0 & 1 & 1 & 0 \\
1 & 0 & 0 & 1
\end{array}\right)\left(\begin{array}{l}
q_{1} \\
q_{2} \\
p_{1} \\
p_{2}
\end{array}\right)
$$


The new co-ordinates $(x, y)$ are again canonical and the semi-simple part $S$ of a quadratic Hamiltonian in 1:-1 resonance becomes

$$
\begin{aligned}
S(x, y) & =\frac{\left(y_{1}-x_{2}\right)^{2}}{4}+\frac{\left(x_{1}+y_{2}\right)^{2}}{4}-\left(\frac{\left(y_{2}-x_{1}\right)^{2}}{4}+\frac{\left(x_{2}+y_{1}\right)^{2}}{4}\right) \\
& =x_{1} y_{2}-x_{2} y_{1}
\end{aligned}
$$

with linear equations of motion

$$
\frac{\mathrm{d}}{\mathrm{d} t}\left(\begin{array}{l}
x_{1} \\
x_{2} \\
y_{1} \\
y_{2}
\end{array}\right)=\left(\begin{array}{cccc}
0 & -1 & 0 & 0 \\
1 & 0 & 0 & 0 \\
0 & 0 & 0 & -1 \\
0 & 0 & 1 & 0
\end{array}\right)\left(\begin{array}{l}
x_{1} \\
x_{2} \\
y_{1} \\
y_{2}
\end{array}\right)
$$

The nilpotent matrix

$$
\left(\begin{array}{llll}
0 & 0 & 0 & 0 \\
0 & 0 & 0 & 0 \\
1 & 0 & 0 & 0 \\
0 & 1 & 0 & 0
\end{array}\right)
$$

has the Hamiltonian function $-N$ where

$$
N(x, y)=\frac{x_{1}^{2}+x_{2}^{2}}{2}
$$

and the Hamiltonian of the transpose matrix is

$$
M(x, y)=\frac{y_{1}^{2}+y_{2}^{2}}{2}
$$

whence $M$ and $N$ are also called each others adjoint.

Exercise 7.5. Show that the two Hamiltonians $S+a N$ and $S-N$ can be obtained from each other by a canonical co-ordinate change if $a<0$. Use in addition reversion of time (i.e. multiply the Hamiltonian by -1 ) to transform $S+N$ into $S-N$.

It is exactly the addition of the adjoint that triggers the Krein collision in the 1-parameter family

$$
H_{2}^{\lambda}(x, y)=S(x, y)+a N(x, y)+\lambda M(x, y)
$$

of quadratic Hamiltonians. Here $a$ is a fixed non-zero coefficient, for example $a=-1$, and $\lambda$ is a parameter passing through 0 .

Exercise 7.6. Compute the eigenvalues $\pm \sqrt{-a \lambda} \pm \mathrm{i}$ of the linear vector field defined by $H_{2}^{\lambda}$. 
The equilibrium is for $a \lambda<0$ of focus-focus type and hence dynamically unstable. At $\lambda=0$ the equilibrium is spectrally stable since no eigenvalue has positive real part. The linear flow is given by

$$
\left(t,\left(\begin{array}{l}
x_{1} \\
x_{2} \\
y_{1} \\
y_{2}
\end{array}\right)\right) \mapsto\left(\begin{array}{cccc}
\cos t & -\sin t & 0 & 0 \\
\sin t & \cos t & 0 & 0 \\
-a t \cos t & a t \sin t & \cos t & -\sin t \\
-a t \sin t & -a t \cos t & \sin t & \cos t
\end{array}\right)\left(\begin{array}{l}
x_{1} \\
x_{2} \\
y_{1} \\
y_{2}
\end{array}\right)
$$

whence the origin is unstable. In Section 7.4 we formulate conditions under which the nonlinear part of an equilibrium in 1:-1 resonance is able to stabilize the equilibrium.

\subsection{Normalization}

We are given a family of Hamiltonian systems for which the origin is an equilibrium, with Taylor series

$$
H^{\lambda}=\sum_{k=0}^{\infty} \frac{H_{k}^{0}}{k !}
$$

where we suppress the parameter dependence in the homogeneous polynomials $H_{k}^{0}$ of degree $k+2$ (the upper index 0 will become clear in a moment). The quadratic part $H_{2}^{\lambda}=\frac{1}{2} H_{0}^{0}$, given by (7.2), admits the symmetry (5.2) and it would be helpful if the higher order terms would respect this symmetry as well. Normal form theory is designed to help in such a situation, pushing a symmetry generated by the quadratic terms through the whole Taylor series.

From an abstract point of view this is an iterative process where at each step a co-ordinate transformation $\varphi_{W}=\varphi_{t=1}^{W}$ is generated by a polynomial Hamiltonian function $W \in \mathbb{R}\left[x_{1}, x_{2}, y_{1}, y_{2}\right]$. Here $W$ is not given to us (like $H$ is), quite on the contrary it is our task is to choose $W$ in such a way that the given Hamiltonian becomes simplified. The effect of $\varphi_{W}$ on $H$ is

$$
H \circ \varphi_{W}=\sum_{k=0}^{\infty} \frac{1}{k !} H_{k}^{0}-\left\{W, H_{0}^{0}\right\}+\sum_{k=1}^{\infty} \frac{1}{k !}\left\{H_{k}^{0}, W\right\}+\ldots
$$

and we concentrate on the effects of lowest order.

Exercise 7.7. Let $A \in M_{n \times n}(\mathbb{R})$ be a matrix for which all eigenvalues are different from each other. Show that the vector space $\mathbb{R}^{n}$ admits the splitting

$$
\operatorname{im} A \oplus \operatorname{ker} A=\mathbb{R}^{n}
$$

as a direct sum of two $A$-invariant subspaces. 
Denoting by $\mathcal{G}_{k+2}$ the polynomials of degree $k+2$, the derivation

$$
\begin{aligned}
& X_{H_{0}^{0}}: \mathcal{G}_{k+2} \longrightarrow \mathcal{G}_{k+2} \\
& W \quad \mapsto \quad\left\{W, H_{0}^{0}\right\}
\end{aligned}
$$

is a linear mapping between finite-dimensional vector spaces. The first step is to choose $W_{1} \in \mathcal{G}_{3}$ such that

$$
\left\{W_{1}, H_{0}^{0}\right\}+H_{0}^{1}=H_{1}^{0}
$$

with $H_{0}^{1}$ in normal form. Then choose $\frac{1}{2} W_{2} \in \mathcal{G}_{4}$ to turn $H_{2}^{0}$ into $H_{0}^{2}$ and so on. At each step the homological equation

$$
\left\{W_{k}, H_{0}^{0}\right\}+H_{0}^{k}=H_{k}^{0}
$$

has to be solved where $\frac{1}{k !} W_{k} \in \mathcal{G}_{k+2}$ generates the $k$ th co-ordinate transformation and $H_{0}^{k} \in \mathcal{G}_{k+2}$ is in normal form (i.e. as simple as possible, preferably zero).

Exercise 7.8. Compute for $H_{0}^{0}=S$ the eigenvalues and eigenvectors of the linear mapping (7.3) and conclude that the splitting

$$
\operatorname{im} X_{S} \oplus \operatorname{ker} X_{S}=\mathcal{G}_{k+2}
$$

can be achieved for every $k \in \mathbb{N}$.

Using the splitting (7.5) the solution of the homological equation (7.4) is immediate; for $H_{k}^{0} \in \mathcal{G}_{k+2}$ we let $H_{0}^{k}$ be the projection to ker $X_{S}$, and since $H_{k}^{0}-H_{0}^{k} \in \operatorname{im} X_{S}$ there must be $W_{k} \in \mathcal{G}_{k+2}$ with $\left\{W_{k}, \mathcal{H}_{0}^{0}\right\}=H_{k}^{0}-H_{0}^{k}$. The infinite composition $\phi_{\infty}=\varphi_{W_{1}} \circ \varphi_{\frac{1}{2} W_{2}} \circ \ldots$ would turn $H$ into the normal form expansion

$$
H \circ \phi_{\infty}=\sum_{k=0}^{\infty} \frac{1}{k !} H_{0}^{k} .
$$

However, the convergence of this normal form series is problematic, and the convergence of the infinite composition is even more questionable. Restricting to a finite composition $\phi_{\ell}=\varphi_{W_{1}} \circ \ldots \circ \varphi_{\frac{1}{\ell !} W_{\ell}}$ yields

$$
H \circ \phi_{\ell}=\bar{H}+\mathcal{O}(\ell+3)
$$

with (truncated) normal form

$$
\bar{H}=\sum_{k=0}^{\ell} \frac{1}{k !} H_{0}^{k}
$$

of degree $\ell+2$ in $x, y$ and higher order terms that are not in normal form. 
Exercise 7.9. Show that the vector space $\mathbb{R}^{n}$ always admits the splitting

$$
\operatorname{im} A \oplus \operatorname{ker} A^{T}=\mathbb{R}^{n}
$$

where $A^{T}$ denotes the transpose of the matrix $A \in M_{n \times n}(\mathbb{R})$.

In the semi-simple case that all eigenvalues have algebraic multiplicity equal to geometric multiplicity (whence one can diagonalise over the complex numbers) there is no difference between the kernel and the kernel of the transpose.

Exercise 7.10. Show that for $H_{0}^{0}=N$ the linear mapping (7.3) is nilpotent, i.e. the power $\left(X_{N}\right)^{m}$ vanishes for sufficiently high $m$ whence all eigenvalues are zero.

Since $X_{S} \circ X_{N}=X_{N} \circ X_{S}$ the sum

$$
X_{H_{0}^{0}}=X_{S}+a X_{N}
$$

is the Jordan decomposition of $X_{H_{0}^{0}}$ into semi-simple and nilpotent part. In an appropriate ${ }^{2}$ basis $X_{H_{0}^{0}}^{T}=X_{S}-a X_{M}$ and

$$
\operatorname{ker} X_{\alpha S-a M}=\operatorname{ker} X_{S} \cap \operatorname{ker} X_{M}
$$

This allows to improve upon the normal form that one would obtain in the semi-simple case $X_{H_{0}^{0}}^{T}=X_{S}$.

Theorem 5.1 implies that every polynomial in $\operatorname{ker} X_{S}$ can be written as a function of $S, N, M$ and

$$
P(x, y)=x_{1} y_{1}+x_{2} y_{2}
$$

Note that this immediately proves $H_{0}^{k}=0$ for all uneven $k$. To compute (7.7) it is sufficient to consider the restriction of $X_{M}$ to the kernel of $X_{S}$ and

$$
\{f, M\}=P \frac{\partial f}{\partial N}+2 M \frac{\partial f}{\partial P}
$$

then shows that polynomials in (7.7) depend only on $S$ and $M$.

Normalizing $H^{\lambda}$ yields (7.6) with $H_{0}^{0}$ unchanged, $H_{0}^{k}=0$ for all uneven $k$ and $H_{0}^{k}=H_{0}^{k}(S, M)$ for all even $k \in \mathbb{N}$, i.e. the normal form reads

$$
\begin{aligned}
\bar{H}^{\lambda}(x, y)= & S(x, y)+a N(x, y)+\lambda M(x, y)+\frac{b}{2}(M(x, y))^{2} \\
& +c S(x, y) M(x, y)+\frac{d}{2}(S(x, y))^{2}+\ldots
\end{aligned}
$$

${ }^{2}$ Alternatively one can introduce an appropriate inner product on $\mathbb{R}\left[x_{1}, x_{2}, y_{1}, y_{2}\right]$ and hence on the vector spaces $\mathcal{G}_{k+2}$. Every orthonormal basis is then 'appropriate'. 
truncated after finitely many monomials in '...'. It turns out that under the non-degeneracy condition $a b \neq 0$ the terms in the second line $(7.8 \mathrm{~b})$ do not influence the qualitative behaviour of the Hamiltonian system defined by (7.8) whence we restrict to the normal form $\bar{H}^{\lambda}$ given by $(7.8 \mathrm{a})$.

Using scalings in $x$ and $y$ and rescaling/reversing time one can simplify the coefficients $a$ and $b$. However, the relative $\operatorname{sign} \operatorname{sgn}(a b)= \pm 1$ cannot be removed and indeed distinguishes two qualitatively different cases. Simplifying to $a=1$ we concentrate on the supercritical case

$$
\bar{H}^{\lambda}=S+N+\lambda M+\frac{1}{2} M^{2}
$$

and leave the subcritical case as exercise 7.14.

\subsection{Symmetry reduction}

The $S^{1}$-symmetry (5.2) generated by $S$ allows to reduce the Hamiltonian system defined by (7.9) to one degree of freedom. This makes $S$ a Casimir function and fixing $S=\mu$ we obtain the reduced phase space

$$
\mathcal{P}_{\mu}=\left\{(N, M, P) \in \mathbb{R}^{3} \mid R_{\mu}(N, M, P)=0, N \geq 0, M \geq 0\right\}
$$

where

$$
R_{\mu}(N, M, P)=\frac{1}{2} P^{2}-2 N M+\frac{\mu^{2}}{2}
$$

with Poisson bracket relations

$$
\{N, M\}=P, \quad\{N, P\}=2 N, \quad\{M, P\}=-2 M .
$$

For $\mu \neq 0$ the surface $\mathcal{P}_{\mu}$ is one sheet of the two-sheeted hyperboloid $R_{\mu}^{-1}(0)$, as in the reduced Kepler system. The surface $\mathcal{P}_{0}$ is the positive cone; in the reduced Kepler system we had to leave out the points reduced from the origin of the configuration space where the potential was not defined. Omitting the constant term the reduced Hamiltonian becomes

$$
\bar{H}_{\mu}^{\lambda}=N+\lambda M+\frac{1}{2} M^{2}
$$

whence the energy level sets $\left\{\bar{H}_{\mu}^{\lambda}=h\right\} \subseteq \mathbb{R}^{3}$ are parabolic cylinders.

Exercise 7.11. Determine the bifurcation diagram of the 3-parameter family

$$
H(x, y)=\frac{1}{2} y^{2}-\frac{1}{5 !} x^{5}+\frac{\nu_{1}}{6} x^{3}-\frac{\nu_{2}}{2} x^{2}-\nu_{3} x
$$

of Hamiltonian systems in one degree of freedom. 


\subsection{The Hamiltonian Hopf bifurcation}

The trajectories of the reduced system are given by the intersections of the energy level sets $\left\{\bar{H}_{\mu}^{\lambda}=h\right\}$ with $\mathcal{P}_{\mu}$. The resulting periodic orbits are organized by the relative equilibria which all lie in the plane $P=0$, cf. Fig. ?. Within this plane the energy level set is the graph of the parabola

$$
N=h-\frac{1}{2} M^{2}-\lambda M
$$

which moves up or down under variation of $h$ and moves to the left or to the right under variation of $\lambda$. The intersection $\mathcal{P}_{\mu} \cap\{P=0\}$ is given by

$$
\begin{array}{rlr}
N=\frac{\mu^{2}}{4 M} & \text { if } \mu \neq 0 \\
N=0 \quad \text { and } \quad M=0 & \text { if } \mu=0 .
\end{array}
$$

Next to the singular equilibrium $(N, M, P)=0$ this yields for $\mu=0$ the regular equilibria $(N, M, P)=(0,-\lambda, 0)$ when $\lambda<0$ and for $\mu \neq 0$ (and all $\lambda)$ the equilibria

$$
(N, M, P)=\left(\frac{\mu^{2}}{4 M}, M, 0\right)
$$

where $M$ is the positive root of the polynomial $M^{3}+\lambda M^{2}-\frac{1}{4} \mu^{2}$.

The full dynamics in $\mathbb{R}^{4}$ of (7.9) is reconstructed by superposing the periodic motion along the angle conjugate to the action $S$. In this way periodic orbits give rise to invariant 2 -tori and regular equilibria yield periodic orbits. The singular equilibrium leads for all $\lambda$ to the equilibrium at the origin which is therefore (dynamically) stable for $\lambda \geq 0$ and unstable for $\lambda<0$. In particular, the linearly unstable equilibrium for $\lambda=0$ has been stablilized by the nonlinear term $\frac{1}{2} M^{2}$. The information on the global dynamics is collected in the energy-momentum mapping.

Exercise 7.12. Show that the critical points of the energy-momentum mapping

$$
\mathcal{E M}=\left(S, \bar{H}^{\lambda}\right): \mathbb{R}^{4} \longrightarrow \mathbb{R}^{2}
$$

form a subset of the discriminant locus of the polynomial

$$
M^{3}+2 \lambda M^{2}-2 h M+\frac{\mu^{2}}{2} .
$$

The discriminant locus of a general polynomial of degree 3 is a cylinder on the basis of the bifurcation diagram derived in exercise 2.8, two smooth surfaces meet at one cusp line. We are interested in the way this set gets folded under the mapping

$$
(\lambda, \mu, h) \mapsto\left(2 \lambda, \frac{\mu^{2}}{2}, 2 h\right)
$$

that assigns to our parameters the coefficients of (7.11). 
Lemma 7.2. The discriminant locus of (7.11) coincides with the discriminant locus of the polynomial

$$
M^{4}-2 \lambda M^{2}+\sqrt{2} \mu M+\frac{h}{2}+\frac{\lambda^{2}}{4} .
$$

To prove this lemma just compute the two discriminant loci. The more interesting question is how to get the idea to compare these two sets. This is much easier to answer after solving exercise 7.11. The bifurcation diagram of (7.10) is the swallow tail surface which lies at the basis of the discriminant locus of the general polynomial of degree 5 (the leading 5 th order term can be scaled to 1 and a translation removes the 4 th order term). With this picture in mind, draw the set of critical values of $\mathcal{E M}$.

Exercise 7.13. Use the translation $M \mapsto M-\frac{2}{3} \lambda$ to turn (7.11) into standard form $M^{3}+\kappa_{1} M+\kappa_{2}$ and study the mapping $\kappa=\kappa(\lambda, \mu, h)$ from $\mathbb{R}^{3}$ to $\mathbb{R}^{2}$.

The mapping

$$
(\lambda, \mu, h) \mapsto\left(\frac{\lambda}{6}, \frac{\sqrt{2} \mu^{2}}{24}, \frac{2 h+\lambda^{2}}{96}\right)
$$

that assigns to our parameters the coefficients of the partial derivative of (7.10) with respect to $x$ is a diffeomorphism whence the discriminant locus of (7.11) is indeed the swallow tail surface. To obtain the set of critical values of the energy-momentum mapping we still have to take the inequalities $N \geq 0$ and $M \geq 0$ into account. The latter is no restriction since the polynomial (7.12) is invariant under the simultaneous transformation $M \mapsto-M, \mu \mapsto-\mu$ and the discriminant locus of (7.12) is symmetric with respect to the reflection in $\mu$. The former inequality yields $h-\frac{1}{2} M^{2}-\lambda M \geq 0$ which results in $h \geq 0$ for $\lambda \leq 0$ and in $h \geq-\frac{1}{2} \lambda^{2}$ for $\lambda \geq 0$. Thus, we have to "remove the tail" from the swallow tail, see Fig. ?.

Note that the $\lambda$-axis consists of critical values of $\mathcal{E M}$ since $S$ and $\bar{H}^{\lambda}$ vanish for all $\lambda$ at the origin. For $\lambda>0$ the critical values form a crease at this line, corresponding to two families of periodic orbits that shrink down to the (stable) equilibrium. For $\lambda<0$ the $\lambda$-axis $\{\mu=0, h=0\}$ detaches from the swallow tail surface and forms a 1 -dimensional thread. The inverse image of these points under the energy-momentum mapping is a pinched torus formed by the unstable equilibrium and its (coinciding) stable and unstable manifold. The thread does belong to the real part of the discriminant locus when interpreting (7.12) as a complex polynomial, but is not part of the (real) discriminant locus when interpreting (7.12) as a real polynomial. Correspondingly, this thread is not part of the bifurcation diagram of (7.10).

Exercise 7.14. Analyse the normal form of the Hamiltonian Hopf bifurcation in the subcritical case $\operatorname{sgn}(a b)=-1$. 
To fully understand the dynamics near a resonant equilibrium in 1:-1 resonance we have to take the higher order terms into account. For higher order terms in normal form one can repeat the above considerations to get precise formulas, but this does not lead to qualitative changes. Indeed, adding higher order terms to a parabola yields a polynomial that still has a local extremum close to the unique extremum of the parabola.

More problematic are higher order terms that are not in normal form. While it is possible to normalize up to any desired degree $\ell+2$ this still leaves us with the remainder term. On an $\varepsilon$-neighbourhood the terms $K$ of order $\mathcal{O}(\ell+3)$ satisfy a bound of the form $\|K\| \leq \varepsilon^{\ell+3} \kappa$ expressing that these terms can be made arbitrarily small. We will see in Chapter 8 that the remainder term nevertheless exerts a certain influence on the invariant 2tori. For the periodic orbits we have the following result, relating the periodic orbits of $H^{\lambda}$ in a neighbourhood of the origin that have a short period ${ }^{3}$ to the periodic orbits of the normal form.

Theorem 7.3. Let $H^{\lambda}$ be a family of Hamiltonian systems on $\mathbb{R}^{4}$ such that for $\lambda=0$ the origin is an equilibrium in 1:-1 resonance, with double eigenvalue $\pm \mathrm{i}$. Assume that the normal form $\bar{H}^{\lambda}$ satisfies

$$
\frac{\partial \bar{H}^{0}}{\partial N}=a \neq 0, \quad \frac{\partial \bar{H}^{\lambda}}{\partial M}=\lambda \quad \text { and } \quad \frac{\partial^{2} \bar{H}^{0}}{\partial M^{2}}=b \neq 0
$$

at $(S, N, M, P)=(0)$. Then there exists a diffeomorphism $\Phi: U \longrightarrow V$ between open neighbourhoods $U, V \subseteq \mathbb{R}^{4}$ of the origin that maps the periodic orbits of $X_{H^{\lambda}}$ with period close to $T=2 \pi$ to the relative equilibria of $X_{\bar{H}^{\lambda}}$.

For a proof see [16]. When $b=0$ both the term $c S M$ in $(7.8 \mathrm{~b})$ and the cubic term in $M$ become important.

\footnotetext{
${ }^{3}$ This excludes the periodic orbits on resonant $2-$ tori.
} 


\section{Perturbation Theory}

The examples of Hamiltonian systems that we considered so far were mostly integrable. In one degree of freedom this follows from conservation of energy, and in two degrees of freedom an existing symmetry allows to reduce to one degree of freedom. Such a symmetry is often only approximate. The assumption of symmetry may be implicitly made when modeling the system, or explicitly introduced by means of a normalization procedure. This makes the behaviour with respect to (small) perturbations an important property of an integrable system.

The recurrent dynamics of an integrable system in two degrees of freedom consists of equilibria, periodic orbits and invariant 2-tori, the former together with stable and unstable manifolds. For equilibria one can use the implicit mapping theorem to prove persistence if the linearization has no zero eigenvalue, and the linear behaviour persists as well if furthermore no eigenvalues are in 1:-1 resonance. Similar considerations are possible for periodic orbits.

The regular values of the energy-momentum mapping of an integrable system parametrise invariant tori (and unbounded motions). Expressed in action-angle variables the Hamiltonian depends only on the actions and this remains true in $n$ degrees of freedom. On the phase space $\mathbb{T}^{n} \times \mathbb{R}^{n}$ with canonical Poisson structure the integrable ${ }^{1}$ system defined by $N(q, p)=N(p)$ has for initial conditions $\left(q_{0}, p_{0}\right)$ the conditionally periodic solution

$$
(q(t), p(t))=\left(q_{0}+\omega\left(p_{0}\right) t, p_{0}\right)
$$

where $\omega(p)=\nabla N(p)$ is the frequency vector of the family of invariant tori $\mathbb{T}^{n} \times\{p\}$. A small perturbation leads to the Hamiltonian function

$$
H_{\varepsilon}(q, p)=N(q)+\varepsilon P(q, p ; \varepsilon) .
$$

By a dictum of Poincaré the study of (8.2) is the fundamental problem of dynamics.

\footnotetext{
${ }^{1}$ It would be more precise to speak here of an integrated system.
} 


\subsection{The homological equation}

The perturbed flow defined by the Hamiltonian (8.2) would be conditionally periodic as well if it were possible to transform co-ordinates $(q, p) \mapsto(x, y)$ in such a way that the resulting $H_{\varepsilon}(x, y)=H_{\varepsilon}(y)$ depends again only on the action variables. We encountered a similar problem when normalizing the higher order terms of the Hamiltonian Hopf bifurcation, so let us try to find a suitable Hamiltonian function $W$ for which the co-ordinate transformation $\varphi_{\varepsilon}=\varphi_{t=\varepsilon}^{W}$ makes the transformed Hamiltonian $H_{\varepsilon} \circ \varphi_{\varepsilon}$ independent of the anglular variables $x \in \mathbb{T}^{n}$. Expanding in $\varepsilon$ yields

$$
\left(H_{\varepsilon} \circ \varphi_{\varepsilon}\right)(x, y)=N(y)+\varepsilon P(x, y ; 0)+\varepsilon\{N, W\}+\mathcal{O}\left(\varepsilon^{2}\right)
$$

whence the terms of order $\varepsilon$ form the homological equation

$$
\left\langle\omega(y) \mid \nabla_{x} W(x, y)\right\rangle+P(y)=P(x, y ; 0)
$$

where we let $P(y)$ denote the $\varepsilon$-term of the transformed Hamiltonian. To solve this linear partial differential equation we develop

$$
\begin{aligned}
W(x, y) & =\sum_{k \in \mathbb{Z}^{n}} W_{k}(y) \mathrm{e}^{2 \pi \mathrm{i}\langle k \mid x\rangle} \\
P(x, y ; 0) & =\sum_{k \in \mathbb{Z}^{n}} P_{k}(y) \mathrm{e}^{2 \pi \mathrm{i}\langle k \mid x\rangle}
\end{aligned}
$$

in Fourier series and are led to $P(y)=P_{0}(y)$ and

$$
\bigwedge_{k \in \mathbb{Z}^{n} \backslash\{0\}} 2 \pi \mathrm{i}\langle k \mid \omega(y)\rangle W_{k}(y)=P_{k}(y)
$$

reflecting the splitting of the function space into $C^{\infty}\left(\mathbb{R}^{n}\right)$ and functions in $C^{\infty}\left(\mathbb{T}^{n} \times \mathbb{R}^{n}\right)$ with vanshing average

$$
P_{0}(y)=\int_{\mathbb{T}^{n}} P(x, y ; 0) \mathrm{d} x .
$$

As the left hand side of (8.4) vanishes at resonances the homological equation cannot be solved in this generality.

Theorem 8.1. The following properties are incompatible.

(i) The unperturbed Hamiltonian is non-degenerate:

$$
\bigwedge_{y} \operatorname{det} D^{2} N(y) \neq 0
$$

(ii) The perturbation $P$ has lots of non-vanishing Fourier coefficients:

$$
\bigwedge_{y} \bigwedge_{k \in \mathbb{Z}^{n}} \bigvee_{\ell \in \mathbb{Z}^{n}} \ell \| k \text { and } P_{\ell}(y) \neq 0 .
$$


(iii) The co-ordinate change $\varphi_{\varepsilon}$ transforms the pertubed Hamiltonian (8.2) into

$$
H(x, y)=N(y)+\varepsilon P_{0}(y)+\varepsilon^{2} R(x, y ; \varepsilon)
$$

with a smaller perturbation term $\varepsilon^{2} R$.

Proof. The first property implies that the frequency mapping $y \mapsto \omega(y)$ is locally invertible and the resonant frequency vectors are dense, so there exists $\eta$ near $y$ and $k \in \mathbb{Z}^{n \backslash\{0\}}$ with $\langle k \mid \omega(\eta)\rangle=0$ and hence $\langle\ell \mid \omega(\eta)\rangle=0$ for all $\ell$ parallel to $k$. Solving (8.4) to obtain the third property then requires

$$
\bigwedge_{\ell \| k} P_{\ell}(\eta)=0
$$

contradicting the second property.

The result goes back to Poincaré, who worked a bit harder and showed that there is no smooth first integral independent of $H_{\varepsilon}$. For a given "unknown" perturbation it is unlikely that lots of Fourier coefficients vanish and hence one should not expect integrability.

\subsection{Small denominators}

Instead of attacking the homological equation in full generality by solving (8.4) simultaneously for all frequencies $\omega(y)$ one can look for simple solutions of the unperturbed system $X_{N}$ and how they survive in the perturbed system $X_{H_{\varepsilon}}$. For the simplest solututions, equilibria and periodic orbits, persistence can be shown by means of the implicit mapping theorem. For quasi-periodic solutions with $n$ independent frequencies the inner product at the right hand side of (8.4) does not vanish, allowing to define the Fourier coefficients

$$
\bigwedge_{k \in \mathbb{Z}^{n} \backslash\{0\}} W_{k}(y)=\frac{P_{k}(y)}{2 \pi \mathrm{i}\left\langle k \mid \omega\left(y_{0}\right)\right\rangle}
$$

of $W$ in terms of a "freezed" frequency vector $\omega=\omega\left(y_{0}\right)$. This yields an exact solution of the homological equation only at $y=y_{0}$, so the approximation of the co-ordinate transformation $\varphi_{\varepsilon}$ is now in terms of both $\varepsilon$ and $\left\|y-y_{0}\right\|$.

From a dynamical point of view it is natural to concentrate on those $n-$ tori $\mathbb{T}^{n} \times\left\{y_{0}\right\}$ with $\langle k \mid \omega\rangle \neq 0$ as these have a dense orbit which makes the torus a dynamically defined object rather than merely an invariant set consisting of quasi-periodic orbits. On the other hand, it is exactly the dense orbit that imposes the denominator $2 \pi \mathrm{i}\langle k \mid \omega\rangle$ to occasionally assume smaller and smaller values. The higher the differentiability of the perturbation $P$ the faster the Fourier coefficients $P_{k}$ converge to zero, and this has to outweigh occuring small values of the denominator if $W$ and the resulting co-ordinate 
transformation are to retain the same degree of differentiability. Thus, one needs bounds, and a $k$-independent bound $|\langle k \mid \omega\rangle|>\gamma$ is available only in the periodic case $n=1$. A typical means to ensure that $|k|=\left|k_{1}\right|+\ldots+\left|k_{n}\right|$ has to be (very) large when $|\langle k \mid \omega\rangle|$ becomes (very) small are Diophantine conditions

$$
\bigwedge_{k \in \mathbb{Z}^{n} \backslash\{0\}}|2 \pi\langle k \mid \omega\rangle| \geq \frac{\gamma}{|k|^{\tau}} .
$$

Exercise 8.1. Sketch the set

$$
\Gamma_{\gamma, \tau}:=\left\{\omega \in \mathbb{R}^{2}|\quad| 2 \pi\langle k \mid \omega\rangle \mid \geq \frac{\gamma}{|k|^{\tau}} \text { for all } k \in \mathbb{Z}^{2 \backslash\{0\}}\right\}
$$

of $(\gamma, \tau)$-Diophantine frequencies.

At first sight the set of $(\gamma, \tau)$-Diophantine frequency vectors seems to be rather small or even empty, as one removes a full open neighbourhood of a dense set. However, by choosing $\tau>n-1$ sufficiently large this set turns out to be of full relative measure in the limit $\gamma \rightarrow 0$.

Exercise 8.2. In the unit interval $[0,1]$, for given constants $\gamma>0$ and $\tau>2$, consider a subset $D_{\gamma, \tau}$ of Diophantine numbers, defined as follows. We say that $\rho \in D_{\gamma, \tau}$ if for all rational numbers $\frac{p}{q}$ one has

$$
\left|\rho-\frac{p}{q}\right| \geq \frac{\gamma}{q^{\tau}}
$$

Show that $D_{\gamma, \tau}$ is nowhere dense. Also show that the Lebesgue measure of $[0,1] \backslash D_{\gamma, \tau}$ is of order $\mathcal{O}(\gamma)$ as $\gamma \rightarrow 0$.

Our aim is to replace the perturbation $P$ by the $\mathbb{T}^{n}$-avarage $P_{0}$. Indeed, the dense orbit on tori with non-resonant $\omega$ is so well distributed that this space average equals the time average along that orbit over infinite time. To have a good approximation over finite time we need that the orbit "visits other parts" of the torus before "coming back" to the starting point.

Apart from this dynamical point of view there is the analytical consideration that the Fourier series (8.3) converges only if the coefficients (8.5) decrease to zero.

Exercise 8.3. Prove the lemma of Paley-Wiener: a periodic function with Fourier series

$$
f(x)=\sum_{k \in \mathbb{Z}} f_{k} \mathrm{e}^{\mathrm{i} k x}
$$

is (real) analytic if and only if the coefficients decay exponentially fast:

$$
\bigvee_{M, \eta>0} \bigwedge_{k \in \mathbb{Z}}\left|f_{k}\right| \leq M \cdot \mathrm{e}^{-|k| \cdot \eta} .
$$


The set of $(\gamma, \tau)$-Diophantine frequency vectors is an example o a Cantor set, intersecting this set $e . g$. with the sphere $\{\|\omega\|=1\}$ transvere to the continuous radial direction yields Cantor dust, a completely disconnected closed set for which every point is an accumulation point (except for a countable subset).

Theorem 8.2. (Kolmogorov, Arnol'd and Moser). Let $\mathbb{Y} \subseteq \mathbb{R}^{n}$ be an open neighbourhood of the origin and consider the phase space $\mathcal{P}=\mathbb{T}^{n} \times \mathbb{Y}$ with canonical Poisson structure. Let the Hamiltonian (8.2) be real analytic with non-degenerate unperturbed part $N$. Then there exists $\varepsilon_{0}>0$ such that for all $|\varepsilon|<\varepsilon_{0}$ there is a canonical transformation $\phi_{\varepsilon}$ near the identity and a measure-theoretically large Cantor set $\mathbb{Y}_{\varepsilon}^{\prime} \subseteq \mathbb{Y}$ with the property that for $y \in \mathbb{Y}_{\varepsilon}^{\prime}$ the transformed Hamiltonian $H_{\varepsilon} \circ \phi_{\varepsilon}$ does not depend on $x \in \mathbb{T}^{n}$.

Since

$$
\begin{aligned}
& \dot{x}=\frac{\partial}{\partial y}\left(H_{\varepsilon} \circ \phi_{\varepsilon}\right)=\widetilde{\omega}(y) \\
& \dot{y}=-\frac{\partial}{\partial x}\left(H_{\varepsilon} \circ \phi_{\varepsilon}\right)=0
\end{aligned}
$$

the Cantor set $\mathbb{T}^{n} \times \mathbb{Y}_{\varepsilon}^{\prime}$ consists of invariant tori with quasi-periodic flow. Note that no statement is made for $y \in \mathbb{Y} \backslash \mathbb{Y}_{\varepsilon}^{\prime}$.

Where the continuous lines of the Cantor set $\mathbb{Y}_{\varepsilon}^{\prime}$ intersect the energy shells transversely we obtain Cantor dust parametrising surviving tori on every energy shell. The same result is obtained under the condition of iso-energetic non-degeneracy

$$
\operatorname{det}\left(\begin{array}{cc}
D^{2} N(y) & \nabla N(y) \\
D N(y) & 0
\end{array}\right) \neq 0,
$$

which is independent of $\operatorname{det} D^{2} N(y) \neq 0$. In two degrees of freedom the energy shells are 3-dimensional whence invariant 2-tori form a barrier. This allows to obtain stability of equilibria (in the sense of Lyapunov).

The (planar) Kepler system does not satisfy a non-degeneracy condition. The same reason that makes the (unperturbed) Keplerian system itself so easy, all reccurent orbits are on ellipses, makes the perturbed problem more difficult. As a remedy one can compute a preliminary normal form

$$
N=K+\varepsilon P_{0}
$$

by averaging the perturbation along the Keplerian flow generated by $X_{K}$. This breaks both the rotational $S^{1}$-symmetry (of any central force field) and the $S^{1}$-symmetry generated by (the direction of) the eccentricity vector. By construction $\{N, K\}=0$, the "Kepler symmetry" makes $N$ integrable, allowing to pass to action angle variables $(\vartheta, \varphi, K, I)$. The iso-energetic non-degeneracy condition

$$
\operatorname{det}\left(\begin{array}{ccc}
\varepsilon \frac{\partial^{2} N}{\partial K^{2}} & \varepsilon \frac{\partial^{2} N}{\partial K \partial I} & 1+\varepsilon \frac{\partial N}{\partial K} \\
\varepsilon \frac{\partial^{2} N}{\partial K \partial I} & \varepsilon \frac{\partial^{2} N}{\partial I^{2}} & \varepsilon \frac{\partial N}{\partial I} \\
1+\varepsilon \frac{\partial N}{\partial K} & \varepsilon \frac{\partial N}{\partial I} & 0
\end{array}\right) \neq 0
$$


is fulfilled if the $\mathcal{O}(\varepsilon)$-term $\varepsilon \frac{\partial^{2} N}{\partial I^{2}}$ does not vanish, in which case the perturbed system has "many" quasi-periodic tori. The two frequencies of these are of order one and of order $\varepsilon$, whence resonances only occur with large values of $k$. 


\section{References}

1. R. Abraham and J.E. Marsden : Foundations of Mechanics, $2^{\text {nd }}$ ed.; Benjamin (1978)

2. V.I. Arnol'd : Mathematical Methods of Classical Mechanics, $2^{\text {nd }}$ ed.; Springer (1989)

3. V.I. Arnol'd and A. Avez : Ergodic problems of classical mechanics ; Benjamin (1968)

4. V.I. Arnol'd, V.V. Kozlov and A.I. Neishtadt : Mathematical Aspects of Classical and Celestial Mechanics ; in Dynamical Systems III (ed. V.I. Arnol'd) Springer (1988)

5. H.W. Broer, F. Dumortier, S.J. van Strien and F. Takens : Structures in dynamics : Finite-dimensional deterministic studies ; Stud. Math. Phys. 2, NorthHolland (1991)

6. H.W. Broer, G.B. Huitema and M.B. Sevryuk: Quasi-Periodic Motions in Families of Dynamical Systems : Order amidst Chaos ; LNM 1645, Springer (1996)

7. R.H. Cushman and L.M. Bates : Global Aspects of Classical Integrable Systems ; Birkhäuser (1997)

8. H. Goldstein : Classical Mechanics ; Addison-Wesley (1980)

9. G. Haller : Chaos near Resonance ; Applied Mathematical Sciences 138, Springer (1999)

10. M.V. Karasev and V.P. Maslov : Nonlinear Poisson Brackets : Geometry and Quantization; Transl. of Mathematical Monographs 119, AMS (1993)

11. L.D. Landau and E.M. Lifshitz : Mechanics ; Course of Theoretical Physics 1, Pergamon (1960)

12. P. Libermann and C.-M. Marle: Symplectic Geometry and Analytical Mechanics ; D. Reidel (1987)

13. R.S. MacKay : Renormalisation in Area-preserving Maps ; World Scientific (1993)

14. J.E. Marsden : Lectures on Mechanics ; Cambridge Univ. Press (1992)

15. J.E. Marsden and T.S. Raţiu : Introduction to Mechanics and Symmetry; Springer (1994)

16. J.C. van der Meer : The Hamiltonian Hopf bifurcation; LNM 1160, Springer (1985) 
17. K.R. Meyer and G.R. Hall : Introduction to Hamiltonian Dynamical Systems and the N-Body Problem; Applied Mathematical Sciences 90, Springer (1992)

18. J. Montaldi and T.S. Raţiu: Geometric Mechanics and Symmetry : the Peyresq Lectures ; Cambridge Univ. Press (2005)

19. J. Moser : Stable and Random Motions in Dynamical Systems : With Special Emphasis on Celestial Mechanics ; Princeton Univ. Press (1973)

20. J. Moser and E.J. Zehnder : Notes on Dynamical Systems ; Courant LNM 12, AMS (2005)

21. P.J. Olver : Applications of Lie groups to differential equations ; Graduate Texts in Mathematics 107, Springer (1986)

22. J.C. Oxtoby: Measure and category. A survey of the analogies between topological and measure spaces; Graduate Texts in Mathematics 2, Springer (1971)

23. V. Poénaru : Singularités $C^{\infty}$ en Présence de Symétrie ; LNM 510, Springer (1976)

24. J.A. Sanders, F. Verhulst and J. Murdock: Averaging Methods in Nonlinear Dynamical Systems, $2^{\text {nd }}$ ed.; Springer (2007)

25. G.W. Schwarz : Smooth functions invariant under the action of a compact Lie group ; Topology 14, p. 63-68 (1975)

26. C.L. Siegel and J.K. Moser : Lectures on Celestial Mechanics ; Springer (1971)

27. F. Verhulst: Nonlinear Differential Equations and Dynamical Systems, $2^{\text {nd }}$ ed.; Springer (1996) 